\title{
Identification of a diffusion coefficient in strongly degenerate parabolic equations with interior degeneracy
}

\author{
Genni Fragnelli ${ }^{1}$, Gabriela Marinoschi ${ }^{2}$, Rosa Maria Mininni ${ }^{1}$ and Silvia \\ Romanelli $^{1}$ \\ ${ }^{1}$ Dipartimento di Matematica, Università degli Studi di Bari Aldo Moro, via \\ E. Orabona 4, 70125 Bari (Italy) \\ ${ }^{2}$ Institute of Mathematical Statistics and Applied Mathematics of the Roma- \\ nian Academy, Calea 13 Septembrie 13, Bucharest (Romania) \\ E-mail: genni.fragnelli@uniba.it,gmarino@acad.ro,rosamaria.mininni@uniba.it \\ and silvia.romanelli@uniba.it
}

\begin{abstract}
We study two identification problems in relation with a strongly degenerate parabolic diffusion equation characterized by a vanishing diffusion coefficient $u \in W^{1, \infty}$, with the property $\frac{1}{u} \notin L^{1}$. The aim is to identify $u$ from certain observations on the solution, by a technique of nonlinear optimal control with control in coefficients. The existence of a controller $u$ which is searched in $W^{1, \infty}$ and the determination of the optimality conditions are given for homogeneous Dirichlet boundary conditions. An approximating problem further introduced allows a better characterization of the optimality conditions, due to the supplementary regularity of the approximating state and dual functions and to a convergence result. Finally, an identification problem with final time observation and homogeneous Dirichlet-Neumann boundary conditions in the state system is considered. By using more technical arguments we provide the explicit form of $u$ and its uniqueness.
\end{abstract}

Keywords: inverse problems, degenerate diffusion equations, internal degeneracy, optimal control, optimality conditions

MSC 2010: 35K65, 35R30, 49N45, 49KXX

\section{Introduction}

In this article we study two identification problems in relation with an evolution equation with a second order differential degenerate operator, in divergence form, $A_{1} y:=\left(u y_{x}\right)_{x}$, when the diffusion coefficient $u$ vanishes at an 
interior point of an one dimensional space domain. The purpose is to determine $u$ from certain observations on the solution to the evolution equation.

Degenerate parabolic operators naturally arise in many problems: BudykoSellers models in climatology (see, e.g., [22]), boundary layer models in physics (see, e.g., [6]), Wright-Fisher and Fleming-Viot models in genetics (see, e.g., [12, 21]), Black-Merton-Scholes models in mathematical finance (see, e.g., [19]). The degenerate operator $A_{1}$ has been studied under different boundary conditions, see, for example, 8], [9], [15], 23]. In [11, [15, the authors consider degenerate operators with boundary conditions of Dirichlet, Neumann, periodic, or nonlinear Robin type. In [1] the authors consider the degenerate operator in divergence and in non divergence form with Dirichlet or Neumann boundary conditions, giving more importance to controllability problems of the associated parabolic evolution equations. However, all previous papers deal with a degenerate operator with degeneracy at the boundary of the domain, for example of the form of the double power function $u(x)=x^{k_{1}}(1-x)^{k_{2}}, x \in[0,1]$, where $k_{1}$ and $k_{2}$ are positive constants.

To the best of our knowledge, Stahel's paper [20] is the first treating a problem with a degeneracy which may be interior. In particular, Stahel considers a parabolic problem in $\mathbb{R}^{N}$ with Dirichlet, Neumann or mixed boundary conditions, associated with a $N \times N$ matrix $a$ (defining the diffusion coefficient), which is positive definite and symmetric, but whose smallest eigenvalue might converge to 0 as the space variable approaches a singular set contained in the closure of the space domain. In this case, he proves that the corresponding abstract Cauchy problem has a solution, provided that $\underline{a}^{-1} \in L^{q}(\Omega, \mathbb{R})$ for some $q>1$, where $\Omega$ is an open bounded subset of $\mathbb{R}^{N}$, and $\underline{a}(x):=\min \{a(x) \xi \cdot \xi:\|\xi\|=1\}$. In [7] there is treated a class of variational degenerate elliptic problems with interior and boundary degeneracy in the case that there exists $k \in(0,2]$ such that $u$ decreases more slowly than $|x-z|^{k}$ near every point $z \in u^{-1}\{0\}$ in the case of bounded domains. The assumption regarding the interior degeneracy of $u$ is generalized in [13 when $N=1$. In particular, in [13] the authors analyze in detail degenerate operators in divergence and non-divergence form, under Dirichlet boundary conditions in spaces $L^{2}(0,1)$ with or without weight and show that under suitable assumptions they generate analytic semigroups. In [14] the same operators with an interior degeneracy are considered, giving more importance to the null controllability of the associated parabolic equations. In [10] a control problem involving a nonlinear nonautonomous operator degenerating on a positive measure interior subset of the space domain is studied. 
The interest in this kind of interior degeneracy problems is due to the fact that they govern diffusion of a substance in water, soil or air, heat flow in a material, diffusion of a population in a habitat. The nonhomogeneity of the medium is expressed by the space dependence of the diffusion coefficient with its possible vanishing at some points. For example, a certain composite material can block the heat flow at a certain point, or the migration of small mammal species can degenerate due to environmental heterogeneity and barriers (see, for example, [5], [16] and the references therein). For this reason it is important to study identification and control problems associated to these degenerate equations. In particular, in [5] and [16] the authors consider two optimal problems: the first one is to minimize the damage and trapping costs, the second one is to maximize the difference between harvesting cost and economic revenue. Another application can be related to the study of the design of biological channels (see, e.g., [18]) in the case when the metabolite diffusion coefficient vanishes.

From the mathematical point of view and in connection with the work of Fragnelli et al. (see [13]) we focus on identifying, on the basis of some observations, the diffusion coefficient in the degenerate parabolic equation

$$
\frac{\partial y}{\partial t}-\frac{\partial}{\partial x}\left(u(x) \frac{\partial y}{\partial x}\right)=f \text { in } Q:=(0, T) \times(0, L), T, L \in(0, \infty),
$$

with the initial condition

$$
y(0, x)=y_{0}(x) \text { in }(0, L),
$$

and various boundary conditions, in particular of homogeneous Dirichlet type

$$
y(t, 0)=y(t, L)=0 \text { in }(0, T) .
$$

We envisage a vanishing $u$ at a point $x_{0} \in(0, L)$ considered in [13] by taking also into account the behavior of the function $\frac{1}{u}$, corresponding either to a so-called slow diffusion (the strongly degenerate case) or to a fast diffusion (the weakly degenerate case).

As far as we know, the particular properties related to the behavior of $\frac{1}{u}$ have not been considered in other identification or control papers.

To motivate our work we specify that the effects of the vanishing diffusion coefficient upon the solution to the diffusion equation (concentration, temperature, density) change according to the particular form of $u(x)$. This 
behavior is illustrated in the graphics realized with Comsol Multiphysics v. 3.5a (FLN License 1025226), e.g., for $u(x)=\left|x-x_{0}\right|^{n}, x \in[0,1], x_{0}=0.5$, $y_{0}=1$. Fig. 1 represents the values of the solution $y(t, x)$ to (1)-(3) along $O x$, for $t=0 ; 0.5 ; 1 ; 1.5 ; 2$, for $n=2$, and Fig. 2 shows the distribution of the values of the solution $y(t, x)$ along $O x$ at the same times for $n=4$. We observe that if $u$ has a zero of a higher order of multiplicity $(n=4)$ at $x_{0}$, then the solution lies at high values in a larger subset of $(0,1)$.

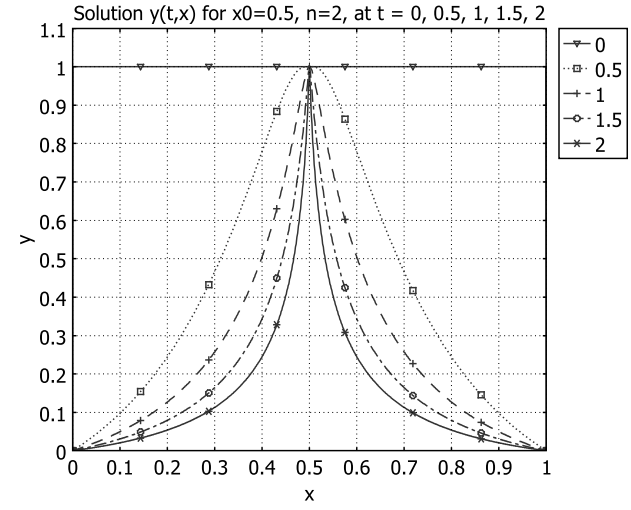

Fig. 1. $y(t, x)$ for $u(x)=\left|x-x_{0}\right|^{2}$

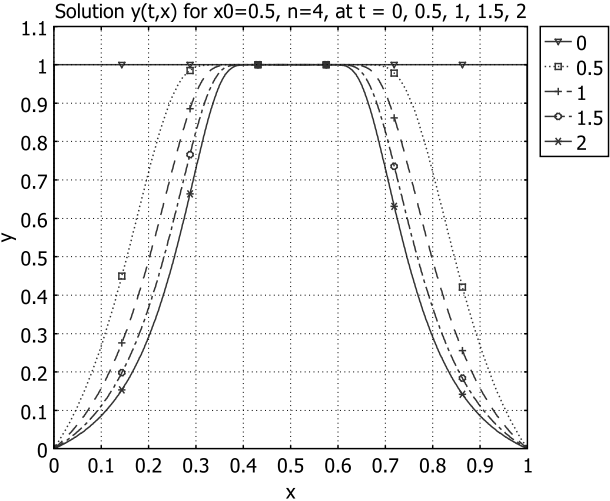

Fig. 2. $y(t, x)$ for $u(x)=\left|x-x_{0}\right|^{4}$

In some practical situations, for instance in a pollutant diffusion process or in a diffusion of a population (bacteria e.g.) in a medium, it is of interest to identify the diffusion coefficient which is suspected to determine high levels of concentration (or density) $y$ in a certain subset of the flow domain due to the possible diffusion stopping at some point $x_{0}$. A similar interest is in the design of a composite material for determining the material properties which preserve the temperature at certain high values.

We mainly aim to determine the function $u(x)$ in the system (11)-(3) from the observation of the spatial mean $\left(M_{T}\right)$ of the state at a final time $T$. However, since other physical quantities, as the mean value $M$ or the mean flux $M_{f}$ over $Q$ may be measured, we shall identify $u$ by combining these possible observations in a unique mathematical problem of minimization of 
a cost functional. Namely, first we study the problem

$$
\begin{aligned}
\text { Minimize } & \left\{\frac{\lambda_{1}}{2}\left(\int_{Q} u(x) y_{x}^{u}(t, x) d x d t-M_{f}\right)^{2}+\frac{\lambda_{2}}{2}\left(\int_{0}^{L} y^{u}(T, x) d x-M_{T}\right)^{2}\right. \\
& \left.+\frac{\lambda_{3}}{2}\left(\int_{Q} y^{u}(t, x) d x d t-M\right)^{2}\right\}
\end{aligned}
$$

subject to (11)-(3), for all $u$ in a set $U$, which will be specified in Section 2.2. Here, $M_{f}, M_{T}, M, \lambda_{1}, \lambda_{2}, \lambda_{3}$, are nonnegative real numbers, and there exists at least one $i \in\{1,2,3\}$ such that $\lambda_{i}>0$.

The notation $y^{u}$ indicates the solution to (11)-(3) corresponding to $u$.

The various choices of the constants $\lambda_{i}, i=1,2,3$, enhance a higher or lower importance to the terms in the functional, according to the problem requirements.

We provide a detailed study of the diffusion equation in the divergence form (11) for the strongly degenerate case (see Definition 2.1) both for homogeneous Dirichlet boundary conditions and for homogeneous DirichletNeumann boundary conditions.

We prove the existence of a solution to the above nonlinear minimization problem, by approaching it as an optimal control problem in coefficients. The diffusion coefficient $u$ is determined by the necessary conditions that $u$, as a solution to $(P)$, should satisfy. They are called optimality conditions. To this end we study the existence in the state system, the system in variations and the dual system associated to the minimization problem. Then we present a detailed computation of the necessary conditions of optimality which is quite technical due to the fact that we search for a function $u$ in $W^{1, \infty}(0, L)$. In the second identification problem we require to identify $u$ from the final observation, imposing to

$$
\text { Minimize }\left\{\int_{0}^{L} y^{u}(T, x) d x\right\}
$$

for all $u \in U$, subject to (11)-(2), with homogeneous Dirichlet-Neumann boundary conditions. In this case we exploit some further properties of the derivatives of the state in order to get a more explicit form of $u$ and its uniqueness. 


\section{Homogeneous Dirichlet boundary conditions}

\subsection{Preliminaries and the state system}

We begin with some notation, definitions and results given in [13], considering the operator $A_{1} y=\left(u y_{x}\right)_{x}$ in the strongly degenerate case. For simplicity we denote $\phi_{t}, \phi_{x}$ the partial derivatives of a generic function $\phi(t, x)$ with respect to $t$ and $x$.

Definition 2.1. $A_{1} y=\left(u y_{x}\right)_{x}$ is called strongly degenerate if there exists $x_{0} \in(0, L)$ such that $u\left(x_{0}\right)=0, u(x)>0$ on $[0, L] \backslash\left\{x_{0}\right\}, u \in W^{1, \infty}(0, L)$ and $\frac{1}{u} \notin L^{1}(0,1)$.

As an example we can mention $u(x)=\left|x-x_{0}\right|^{k}, k \geq 1$.

We define the weighted space

$$
\begin{aligned}
H_{u}^{1}(0, L) & =\left\{y \in L^{2}(0, L) ; y \text { locally absolutely continuous in }[0, L] \backslash\left\{x_{0}\right\},\right. \\
\sqrt{u} y_{x} & \left.\in L^{2}(0, L), y(0)=y(L)=0\right\},
\end{aligned}
$$

with the norm

$$
\|y\|_{H_{u}^{1}(0, L)}=\left(\|y\|_{L^{2}(0, L)}^{2}+\left\|\sqrt{u} y_{x}\right\|_{L^{2}(0, L)}^{2}\right)^{1 / 2} .
$$

According to [13], Proposition 2.3, we have that

$$
\begin{gathered}
H_{u}^{1}(0, L)=\left\{y \in L^{2}(0, L) ; y \text { locally absolutely continuous in }[0, L] \backslash\left\{x_{0}\right\},\right. \\
\left.\sqrt{u} y_{x} \in L^{2}(0, L), u y \text { is continuous at } x_{0}, y(0)=y(L)=(u y)\left(x_{0}\right)=0\right\} .
\end{gathered}
$$

We specify that $H_{u}^{1}(0, L)$ is a Hilbert space and $H_{u}^{1}(0, L) \hookrightarrow L^{2}(0, L) \hookrightarrow$ $\left(H_{u}^{1}(0, L)\right)^{\prime}$, where $\left(H_{u}^{1}(0, L)\right)^{\prime}$ is the dual of $H_{u}^{1}(0, L)$ and "ฯ" means a continuous and dense embedding.

For simplicity, we denote

$$
H=L^{2}(0, L), V_{u}=H_{u}^{1}(0, L), V_{u}^{\prime}=\left(H_{u}^{1}(0, L)\right)^{\prime},
$$

where they indicate subscripts.

Let us consider

$$
H_{u}^{2}(0, L)=\left\{y \in H_{u}^{1}(0, L) ; u y_{x} \in H^{1}(0, L)\right\}
$$


and define

$$
A_{1} y:=\left(u y_{x}\right)_{x}, A_{1}: D\left(A_{1}\right) \subset L^{2}(0, L) \rightarrow L^{2}(0, L), D\left(A_{1}\right)=H_{u}^{2}(0, L) .
$$

According to [13], Proposition 2.4,

$$
\begin{aligned}
D\left(A_{1}\right) & =\left\{y \in L^{2}(0, L) ; y \text { is absolutely continuous in }[0, L] \backslash\left\{x_{0}\right\}\right. \\
u y & \left.\in H_{0}^{1}(0, L), u y_{x} \in H^{1}(0, L), u y\left(x_{0}\right)=u y_{x}\left(x_{0}\right)=0\right\}
\end{aligned}
$$

By [13, Theorem 2.7, $A_{1}: D\left(A_{1}\right) \rightarrow L^{2}(0, L)$ is self-adjoint, nonpositive on $L^{2}(0, L)$ and it generates a positivity preserving semigroup. This result is used further to prove that (1) -(3) has a unique mild solution if $y_{0} \in L^{2}(0, L)$ and $f \in L^{2}(Q)$. This is a strong solution if $y_{0} \in H_{u}^{2}(0, L)$ and also if $y_{0} \in$ $H_{u}^{1}(0, L)$ (see Theorem 4.1 and Remark 4.2 in [13]).

We also recall the following result (Lemma 2.6 in [13]):

Lemma 2.2. For all $(y, z) \in H_{u}^{2}(0, L) \times H_{u}^{1}(0, L)$ one has

$$
\int_{0}^{L}\left(u y_{x}\right)_{x} z d x=-\int_{0}^{L} u y_{x} z_{x} d x
$$

For the purposes of our paper we present the existence result for the state system (1)-(3) by a variational way. For convenience, and where no confusion can be made we shall not write the function arguments in the integrands.

Definition 2.3. Let $y_{0} \in L^{2}(0, L), f \in L^{2}\left(0, T ;\left(H_{u}^{1}(0, L)\right)^{\prime}\right)$ and $u$ with the properties of Definition 2.1. We call a solution to (11)-(3) a function

$$
y \in C\left([0, T] ; L^{2}(0, L)\right) \cap L^{2}\left(0, T ; H_{u}^{1}(0, L)\right) \cap W^{1,2}\left([0, T] ;\left(H_{u}^{1}(0, L)\right)^{\prime}\right),
$$

which satisfies the equation

$$
\int_{0}^{T}\left\langle\frac{d y}{d t}(t), \psi(t)\right\rangle_{V_{u}^{\prime}, V_{u}} d t+\int_{Q} u y_{x} \psi_{x} d x d t=\int_{0}^{T}\langle f(t), \psi(t)\rangle_{V_{u}^{\prime}, V_{u}} d t
$$

for any $\psi \in L^{2}\left(0, T ; H_{u}^{1}(0, L)\right)$, and the initial condition $y(0)=y_{0}$.

Theorem 2.4. If $y_{0} \in L^{2}(0, L), f \in L^{2}\left(0, T ;\left(H_{u}^{1}(0, L)\right)^{\prime}\right)$, then (11)-(13) has a unique solution

$$
y \in C\left([0, T] ; L^{2}(0, L)\right) \cap L^{2}\left(0, T ; H_{u}^{1}(0, L)\right) \cap W^{1,2}\left([0, T] ;\left(H_{u}^{1}(0, L)\right)^{\prime}\right),
$$


satisfying the estimate

$$
\sup _{t \in[0, T]}\|y(t)\|_{H}^{2}+\int_{0}^{T}\|y(t)\|_{V_{u}}^{2} d t \leq C_{T}\left(\left\|y_{0}\right\|_{H}^{2}+\|f\|_{L^{2}\left(0, T ; V_{u}^{\prime}\right)}^{2}\right) .
$$

If, in addition $y_{0} \in H_{u}^{1}(0, L)$ and $f \in L^{2}(Q)$, then

$$
y \in W^{1,2}\left([0, T] ; L^{2}(0, L)\right) \cap L^{2}\left(0, T ; H_{u}^{2}(0, L)\right) \cap L^{\infty}\left(0, T ; H_{u}^{1}(0, L)\right)
$$

and it satisfies

$$
\sup _{t \in[0, T]}\|y(t)\|_{V_{u}}^{2}+\int_{0}^{T}\left(\left\|\frac{d y}{d t}(t)\right\|_{H}^{2}+\left\|\left(u y_{x}\right)_{x}(t)\right\|_{H}^{2}\right) d t \leq C_{T}\left(\left\|y_{0}\right\|_{V_{u}}^{2}+\|f\|_{L^{2}(Q)}^{2}\right)
$$

where $C_{T}$ denotes several positive constants.

If $y_{0} \geq 0$ a.e. on $(0, L)$ and $f \geq 0$ a.e. on $Q$, then $y(t) \geq 0$ a.e. in $(0, L)$ for all $t \in[0, T]$.

Proof. Let us introduce the linear operator

$$
A: H_{u}^{1}(0, L) \rightarrow\left(H_{u}^{1}(0, L)\right)^{\prime}
$$

by

$$
\langle A z, \psi\rangle_{V_{u}^{\prime}, V_{u}}=\int_{0}^{L} u(x) z_{x}(x) \psi_{x}(x) d x, \text { for any } \psi \in H_{u}^{1}(0, L) .
$$

It is continuous and monotone

$$
\begin{gathered}
\|A z\|_{V_{u}^{\prime}}=\sup _{\psi \in V_{u},\|\psi\|_{V_{u}} \leq 1}\left|\langle A z, \psi\rangle_{V_{u}^{\prime}, V_{u}}\right| \leq\|z\|_{V_{u}}, \\
\langle A z, z\rangle_{V_{u}^{\prime}, V_{u}} \geq 0
\end{gathered}
$$

and has the property

$$
\langle A z, z\rangle_{V_{u}^{\prime}, V_{u}}=\int_{0}^{L} u z_{x}^{2} d x=\|z\|_{V_{u}}^{2}-\|z\|_{H}^{2} .
$$

We consider the Cauchy problem

$$
\begin{aligned}
\frac{d y}{d t}(t)+A y(t) & =f(t), \text { a.e. } t \in(0, T) \\
y(0) & =y_{0} .
\end{aligned}
$$




$$
y_{0} \in L^{2}(0, L), f \in L^{2}\left(0, T ;\left(H_{u}^{1}(0, L)\right)^{\prime}\right)
$$

then the Cauchy problem has a unique solution belonging to the spaces indicated in (9) (see e.g. [17, p. 162). Obviously, this is a solution to (1)-(3) in the sense of Definition 2.3, so that (10) and (18) are equivalent. The estimate (11) follows by setting in (10) $\psi=y$ and performing a few computations involving the Gronwall's lemma.

Now, we observe that $-A_{1}$ is the restriction of $A$ on $L^{2}(0, L)$. Indeed, if $y \in$ $H_{u}^{2}(0, L)$, by (8) we see that $-A_{1}$ coincides with $A$. Therefore, if $y_{0} \in D\left(A_{1}\right)=$ $H_{u}^{2}(0, L)$ and $f \in W^{1,1}\left([0, T] ; L^{2}(0, L)\right)$, problem (18) with $A$ replaced by its restriction $-A_{1}$ has a more regular solution $y \in W^{1, \infty}\left([0, T] ; L^{2}(0, L)\right) \cap$ $L^{\infty}\left(0, T ; H_{u}^{2}(0, L)\right)$. This follows by Theorem 4.9 (see [3, p. 151). If $y_{0} \in$ $H_{u}^{1}(0, L)$ and $f \in L^{2}(Q)$, the density of $H_{u}^{2}(0, L)$ in $H_{u}^{1}(0, L)$, the density of $W^{1,1}\left([0, T] ; L^{2}(0, L)\right)$ in $L^{2}(Q)$ and some standard estimates lead to the second part of the theorem.

Finally, the nonnegativity of the solution follows by the positivity preserving property of the semigroup generated by $A_{1}$.

\subsection{Existence in $(P)$}

We denote

$$
\begin{aligned}
J(u)= & \frac{\lambda_{1}}{2}\left(\int_{Q} u y_{x}^{u} d x d t-M_{f}\right)^{2}+\frac{\lambda_{2}}{2}\left(\int_{0}^{L} y^{u}(T, x) d x-M_{T}\right)^{2} \\
& +\frac{\lambda_{3}}{2}\left(\int_{Q} y^{u} d x d t-M\right)^{2}
\end{aligned}
$$

and introduce the minimization problem

$$
\text { Minimize } J(u) \text { for all } u \in U,
$$

subject to (11)-(3), where

$$
\begin{aligned}
U & =\left\{u \in W^{1, \infty}(0, L) ; u_{m}(x) \leq u(x) \leq u_{M}(x),\right. \\
u(0) & \left.=u_{0}, u(L)=u_{L},\left|u_{x}(x)\right| \leq u_{\infty} \text { a.e. } x \in(0, L)\right\} .
\end{aligned}
$$

We assume the following hypotheses:

$$
\begin{aligned}
x_{0} & \in(0, L), u_{\infty} \in[0, \infty), \\
u_{m}, u_{M} & \in C[0, L], u_{M}(x) \leq \alpha(x) u_{m}(x) \text { for } x \in[0, L], \alpha \in C[0, L], \alpha \geq 1, \\
0 & <u_{m}(x)<u_{M}(x) \text { for } x \in[0, L] \backslash\left\{x_{0}\right\}, u_{m}\left(x_{0}\right)=u_{M}\left(x_{0}\right)=0,
\end{aligned}
$$


and

$$
\int_{0}^{L} \frac{1}{u_{M}(x)} d x=+\infty .
$$

Then, by (21), for all $u \in U$ we have

$$
0<u_{m}(0) \leq u_{0} \leq u_{M}(0), 0<u_{m}(L) \leq u_{L} \leq u_{M}(L)
$$

These conditions ensure the fact that the operator $A_{1}$ is strongly degenerate, because $u \in U$ and (21) imply that $u\left(x_{0}\right)=0, u>0$ in $[0, L] \backslash\left\{x_{0}\right\}$, and (22) establishes that $\frac{1}{u} \notin L^{1}(0, L)$.

Moreover, the assumption $u_{M}(x) \leq \alpha(x) u_{m}(x)$ implies that if $u, v \in U$ then $\left|\frac{v}{u}(x)\right| \leq\|\alpha\|_{L^{\infty}(0, L)}$ for $x \in[0, L] \backslash\left\{x_{0}\right\}$ and

$$
H_{u}^{1}(0, L)=H_{v}^{1}(0, L) \text { for any } u, v \in U .
$$

Indeed, if $y \in H_{u}^{1}(0, L)$ then $y$ is locally absolutely continuous in $[0, L] \backslash\left\{x_{0}\right\}$, $y \in L^{2}(0, L), \sqrt{u} y_{x} \in L^{2}(0, L), y(0)=y(L)=0$. Let $v \in U$. Then, by a simple calculation

$$
\int_{0}^{L} v y_{x}^{2} d x \leq\|\alpha\|_{L^{\infty}(0, L)}\left\|\sqrt{u} y_{x}\right\|_{L^{2}(0, L)}^{2}
$$

and so $y \in H_{v}^{1}(0, L)$. Analogously, we get $H_{v}^{1}(0, L) \subset H_{u}^{1}(0, L)$.

Theorem 2.5. Let $y_{0} \in L^{2}(0, L), y_{0} \geq 0$ on $(0, L), f \in L^{2}(Q), f \geq 0$ a.e. on $Q$. Then $(P)$ has at least one solution $u$ with the corresponding state

$$
y \in C\left([0, T] ; L^{2}(0, L)\right) \cap L^{2}\left(0, T ; H_{u}^{1}(0, L)\right) \cap W^{1,2}\left([0, T] ;\left(H_{u}^{1}(0, L)\right)^{\prime}\right) .
$$

If $y_{0} \in H_{u}^{1}(0, L)$, then the state $y$ is more regular, as in (12).

Proof. Under the specified hypotheses, problem (11)-(3) has a unique nonnegative solution given by Theorem 2.4. Then, $J(u) \geq 0$, its infimum exists and it is nonnegative. Let us denote it by $d$.

For not overloading the notations we shall drop the superscript ${ }^{u}$.

We consider a minimizing sequence $\left(u_{n}\right)_{n \geq 1}, u_{n} \in U$ which satisfies

$$
\begin{aligned}
d \leq & \frac{\lambda_{1}}{2}\left(\int_{Q} u_{n} y_{n x} d x d t-M_{f}\right)^{2}+\frac{\lambda_{2}}{2}\left(\int_{0}^{L} y_{n}(T, x) d x-M_{T}\right)^{2} \\
& +\frac{\lambda_{3}}{2}\left(\int_{Q} y_{n} d x d t-M\right)^{2} \leq d+\frac{1}{n}
\end{aligned}
$$


where the corresponding state $y_{n}$ is the solution to (11)-(3) (equivalently to (18)) with $u$ replaced by $u_{n}$. By Theorem 2.4, it exists for each $n$, it is unique and satisfies

$$
\sup _{t \in[0, T]}\left\|y_{n}(t)\right\|_{H}^{2}+\int_{0}^{T}\left\|y_{n}(t)\right\|_{V_{u_{n}}}^{2} d t \leq C, \text { for any } t \in[0, T],
$$

with $C$ a positive constant independent of $n$, by (13).

Since $u_{n} \in U$, we deduce that there exist subsequences (denoted still by the subscript ${ }_{n}$ ) such that

$$
\begin{gathered}
u_{n} \rightarrow u \text { weak }{ }^{*} \text { in } L^{\infty}(0, L), \text { as } n \rightarrow \infty, \\
u_{n x} \rightarrow u_{x} \text { weak }^{*} \text { in } L^{\infty}(0, L), \text { as } n \rightarrow \infty, \\
y_{n} \rightarrow y \text { weak* in } L^{\infty}\left(0, T ; L^{2}(0, L)\right) \text {, as } n \rightarrow \infty, \\
y_{n}(T) \rightarrow \zeta \text { weakly in } L^{2}(0, L), \text { as } n \rightarrow \infty .
\end{gathered}
$$

By (27), $\left(\sqrt{u_{n}} y_{n x}\right)_{n}$ is bounded in $L^{2}(Q)$ and there exists $\xi \in L^{2}(Q)$ such that, on a subsequence (denoted still by the subscript ${ }_{n}$ )

$$
\xi_{n}:=\sqrt{u_{n}} y_{n x} \rightarrow \xi \text { weakly in } L^{2}(Q) \text {, as } n \rightarrow \infty \text {. }
$$

Let $\delta$ be positive, arbitrary. Then $\xi_{n} \rightarrow \xi$ weakly in $L^{2}\left(0, T ; L^{2}\left(\Omega_{\delta}\right)\right)$ too, where $\Omega_{\delta}=\left(0, x_{0}-\delta\right) \cup\left(x_{0}+\delta, L\right)$.

The previous first two convergences for $u_{n}$ imply that

$$
u_{n} \rightarrow u \text { uniformly on }[0, L] \text {, as } n \rightarrow \infty \text {, }
$$

and therefore the sequence $y_{n x}=\frac{\xi_{n}}{\sqrt{u_{n}}}$ is bounded in $L^{2}\left(0, T ; L^{2}\left(\Omega_{\delta}\right)\right)$ and

$$
y_{n x}=\frac{\xi_{n}}{\sqrt{u_{n}}} \rightarrow \frac{\xi}{\sqrt{u}} \text { weakly in } L^{2}\left(0, T ; L^{2}\left(\Omega_{\delta}\right)\right) \text {, as } n \rightarrow \infty \text {. }
$$

On the other hand, $y_{n x} \rightarrow y_{x}$ in the sense of distributions. We conclude that $\xi=\sqrt{u} y_{x}$ a.e. on $(0, T) \times \Omega_{\delta}$ and since $\delta$ is arbitrary we finally get $\xi=\sqrt{u} y_{x}$ a.e. on $Q$. It follows that $y \in L^{2}\left(0, T ; H_{u}^{1}(0, L)\right)$ and so

$$
y_{n} \rightarrow y \text { weakly in } L^{2}\left(0, T ; H_{u}^{1}(0, L)\right) \text {, as } n \rightarrow \infty \text {. }
$$

Next, by the definition of $A$, see (14), we have that

$$
A y_{n} \rightarrow A y \text { weakly in } L^{2}\left(0, T ;\left(H_{u}^{1}(0, L)\right)^{\prime}\right) \text {, as } n \rightarrow \infty \text {, }
$$


and by (18) we deduce that $\left(\frac{d y_{n}}{d t}\right)_{n}$ is bounded in $L^{2}\left(0, T ;\left(H_{u}^{1}(0, L)\right)^{\prime}\right)$, so that by selecting a subsequence we have

$$
\frac{d y_{n}}{d t} \rightarrow \frac{d y}{d t} \text { weakly in } L^{2}\left(0, T ;\left(H_{u}^{1}(0, L)\right)^{\prime}\right) \text {, as } n \rightarrow \infty .
$$

Moreover, since $y_{n}$ is the solution to (18) we get that

$$
\begin{aligned}
y_{n}(t) & =y_{0}+\int_{0}^{t}\left(-A y_{n}(s)+f(s)\right) d s \\
& \rightarrow y_{0}+\int_{0}^{t}(-A y(s)+f(s)) d s, \text { weakly in }\left(H_{u}^{1}(0, L)\right)^{\prime}, \text { for all } t \in[0, T] .
\end{aligned}
$$

From here we get that

$$
\int_{0}^{L} y_{n}(t) \phi_{0} d x=\int_{0}^{L} y_{0} \phi_{0} d x+\int_{0}^{t}\left\langle-A y_{n}(s)+f(s), \phi_{0}\right\rangle_{V_{u}^{\prime}, V_{u}} d s
$$

for any $\phi_{0} \in H_{u}^{1}(0, L)$ and $t \in[0, T]$. Passing to the limit we obtain

$$
l(t)=\lim _{n \rightarrow \infty} \int_{0}^{L} y_{n}(t) \phi_{0} d x=\int_{0}^{L} y_{0} \phi_{0} d x+\int_{0}^{t}\left\langle-A y(s)+f(s), \phi_{0}\right\rangle_{V_{u}^{\prime}, V_{u}} d s .
$$

We multiply this relation by $\phi_{1} \in L^{2}(0, T)$ and integrate over $(0, T)$, getting

$$
\int_{0}^{T} \phi_{1}(t) l(t) d t=\int_{0}^{T}\left(\int_{0}^{L} y_{0} \phi_{0} d x+\int_{0}^{t}\left\langle-A y(s)+f(s), \phi_{0}\right\rangle_{V_{u}^{\prime}, V_{u}} d s\right) \phi_{1}(t) d t .
$$

We multiply (28) by $\phi_{1}(t) \phi_{0}(x)$ and integrate over $(0, T) \times(0, L)$,

$$
\int_{Q} \phi_{0} \phi_{1} y_{n} d x d t=\int_{0}^{T}\left(\int_{0}^{L} y_{0} \phi_{0} d x+\int_{0}^{t}\left\langle-A y_{n}(s)+f(s), \phi_{0}\right\rangle_{V_{u}^{\prime}, V_{u}} d s\right) \phi_{1}(t) d t .
$$

By the weak convergence $y_{n} \rightarrow y$ in $L^{2}(Q)$ we get that

$$
\int_{Q} \phi_{0} \phi_{1} y d x d t=\int_{0}^{T}\left(\int_{0}^{L} y_{0} \phi_{0} d x+\int_{0}^{t}\left\langle-A y(s)+f(s), \phi_{0}\right\rangle_{V_{u}^{\prime}, V_{u}} d s\right) \phi_{1}(t) d t .
$$

Comparing (30) and (32) we deduce that

$$
\int_{0}^{T} \phi_{1}(t) l(t) d t=\int_{Q} \phi_{0} \phi_{1} y d x d t, \text { for any } \phi_{0} \in H_{u}^{1}(0, L), \phi_{1} \in L^{2}(0, T),
$$


hence

$$
l(t)=\lim _{n \rightarrow \infty} \int_{0}^{L} y_{n}(t) \phi_{0} d x=\int_{0}^{L} y(t) \phi_{0} d x, \text { for any } \phi_{0} \in H_{u}^{1}(0, L), t \in[0, T] .
$$

Therefore,

$$
y_{0}=y_{n}(0) \rightarrow y(0), y_{n}(T) \rightarrow y(T) \text { weakly in }\left(H_{u}^{1}(0, L)\right)^{\prime}, \text { as } n \rightarrow \infty
$$

and so by the limit uniqueness $\zeta=y(T)$ and $y(0)=y_{0}$ a.e. on $(0, L)$.

Now, $y_{n}$ satisfies (10)

$$
\int_{0}^{T}\left\langle\frac{d y_{n}}{d t}(t), \psi(t)\right\rangle_{V_{u}^{\prime}, V_{u}} d t+\int_{Q} u_{n} y_{n x} \psi_{x} d x d t=\int_{Q} f \psi d x d t
$$

for any $\psi \in L^{2}\left(0, T ; H_{u}^{1}(0, L)\right)$ and passing to the limit as $n \rightarrow \infty$ we get that $y$ satisfies (10), too. All these assertions prove that $y$ is the solution to (11)-(3) corresponding to $u$.

Next, if $y_{0} \in H_{u}^{1}(0, L)$, the solution $y$ previously obtained has the regularity (12) according to Theorem 2.4.

Finally, we pass to the limit in (26) as $n \rightarrow \infty$, on the basis of the weakly lower semicontinuity of each convex term in $J\left(u_{n}\right)$, and get that $d=J(u)$.

Since $U$ is closed, then $u(x) \in\left[u_{m}(x), u_{M}(x)\right]$ which implies by (21) and (22) that $u(x)>0$ on $[0, L] \backslash\left\{x_{0}\right\}, u\left(x_{0}\right)=0$ and $\frac{1}{u} \notin L^{1}(0, L)$, so that $u \in U$ and the corresponding operator $A_{1} y=\left(u y_{x}\right)_{x}$ is strongly degenerate.

\subsection{Optimality conditions in the homogeneous Dirich- let case}

Proposition 2.6. Let $\left(u^{*}, y^{*}\right)$ be a solution to $(P)$. Then $u^{*}$ satisfies the necessary condition

$$
\int_{Q}\left(u^{*}-u\right) y_{x}^{*}\left[p_{x}+\lambda_{1}\left(\int_{Q} u^{*} y_{x}^{*} d x d t-M_{f}\right)\right] d x d t \leq 0
$$

for all $u \in U$, where $p$ is the solution to

$$
\begin{aligned}
& \frac{\partial p}{\partial t}+\left(u^{*} p_{x}\right)_{x} \\
= & -\lambda_{1}\left(\int_{Q} u^{*} y_{x}^{*} d x d t-M_{f}\right)+\lambda_{3}\left(\int_{Q} y^{*} d x d t-M\right) \text { in } Q,
\end{aligned}
$$




$$
\begin{gathered}
p(T, x)=-\lambda_{2}\left(\int_{0}^{L} y^{*}(T, x) d x-M_{T}\right) \text { in }(0, L), \\
p(t, 0)=p(t, L)=0 \text { in }(0, T) .
\end{gathered}
$$

Proof. Let $\left(u^{*}, y^{*}\right)$ be a solution to $(P), \lambda>0, u \in U$ and denote

$$
u^{\lambda}(x)=u^{*}(x)+\lambda v(x),
$$

where

$$
v(x)=u(x)-u^{*}(x), u \in U .
$$

It is obvious that $v \in W^{1, \infty}(0, L), v\left(x_{0}\right)=0, u_{m}(x)-u_{M}(x) \leq v(x) \leq u(x)$, $v(0)=v(L)=0$ and $\frac{1}{v} \notin L^{1}(0, L)$. We introduce the system

$$
\begin{gathered}
\frac{\partial Y}{\partial t}-\left(u^{*} Y_{x}\right)_{x}=\left(v y_{x}^{*}\right)_{x} \text { in } Q, \\
Y(0, x)=0 \text { in }(0, L), \\
Y(t, 0)=Y(t, L)=0 \text { in }(0, T) .
\end{gathered}
$$

We note that since $y^{*} \in L^{2}\left(0, T ; H_{u^{*}}^{1}(0, L)\right)$, after a few calculations it follows that

$$
\int_{Q} v^{2}\left(y_{x}^{*}\right)^{2} d x d t \leq\left\|u_{M}\right\|_{C[0, L]}\|\alpha\|_{L^{\infty}(0, L)} \int_{0}^{T}\left\|\sqrt{u^{*}} y_{x}^{*}(t)\right\|_{H}^{2} d t
$$

Hence, $v y_{x}^{*} \in L^{2}\left(0, T ; L^{2}(0, L)\right)$ and $g=\left(v y_{x}^{*}\right)_{x} \in L^{2}\left(0, T ;\left(H_{u^{*}}^{1}(0, L)\right)^{\prime}\right)$, since for any $\psi \in H_{u^{*}}^{1}(0, L)$, and a.e. $t \in(0, T)$ we have

$$
\left|\left\langle\left(v y_{x}^{*}\right)_{x}(t), \psi\right\rangle_{V_{u^{*}}^{\prime}, V_{u^{*}}}\right|=\left|-\int_{0}^{L} v y_{x}^{*}(t) \psi_{x} d x\right| \leq\|\alpha\|_{L^{\infty}(0, L)}\left\|y^{*}(t)\right\|_{V_{u^{*}}}\|\psi\|_{V_{u^{*}}} .
$$

We state that (38)-(40) has a unique solution

$$
Y \in C\left([0, T] ; L^{2}(0, L)\right) \cap L^{2}\left(0, T ; V_{u^{*}}\right) \cap W^{1,2}\left([0, T] ; V_{u^{*}}^{\prime}\right) .
$$

This follows as in the first part of Theorem 2.4 by defining the operator $A$ from $H_{u^{*}}^{1}(0, L)$ to $\left(H_{u^{*}}^{1}(0, L)\right)^{\prime}$, by

$$
\langle A z, \psi\rangle_{V_{u^{*}}^{\prime}, V_{u^{*}}}=\int_{0}^{L} u^{*} z_{x} \psi_{x} d x, \text { for any } \psi \in V_{u^{*}}
$$


Moreover, denoting by $y^{\lambda}(t, x)$ the solution to (11)-(3) corresponding to $u^{\lambda}(x)$, one can prove that actually

$$
Y(t, x)=\lim _{\lambda \rightarrow 0} \frac{y^{\lambda}(t, x)-y^{*}(t, x)}{\lambda},
$$

so that (38)-(40) is the system of first order variations.

We introduce the dual system (34)-(36). This system has a unique solution

$$
\begin{array}{r}
p \in C\left([0, T] ; H_{u^{*}}^{1}(0, L)\right) \cap L^{2}\left(0, T ; H_{u^{*}}^{2}(0, L)\right) \cap W^{1,2}\left([0, T] ; L^{2}(0, L)\right), \\
\left(u^{*} p_{x}\right)_{x} \in L^{2}(Q),
\end{array}
$$

given still by Theorem 2.4, second part (after making the transformation $\left.t^{\prime}=T-t\right)$.

Now, we write that $\left(u^{*}, y^{*}\right)$ is a solution to $(P)$, that is

$$
J\left(u^{*}\right) \leq J(u) \text {, for all } u \in U,
$$

and, in particular, for $u=u^{\lambda}$. After some algebra we get

$$
\lambda_{1} I_{f} \int_{Q}\left(-u_{x}^{*} Y+v y_{x}^{*}\right) d x d t+\lambda_{2} I_{T} \int_{0}^{L} Y(T, x) d x+\lambda_{3} I_{M} \int_{Q} Y d x d t \geq 0,
$$

where

$$
I_{f}=\int_{Q} u^{*} y_{x}^{*} d x d t-M_{f}, I_{T}=\int_{0}^{L} y^{*}(T, x) d x-M_{T}, I_{M}=\int_{Q} y^{*} d x d t-M .
$$

We test (38) by $p(t)$ and integrate over $(0, T)$. After some calculations we get

$$
\begin{aligned}
& \int_{Q}-\left(p_{t}+\left(u^{*}(x) p_{x}\right)_{x}\right) Y d x d t+\int_{0}^{L} p(T, x) Y(T, x) d x \\
= & \int_{0}^{T}\left\langle\left(v y_{x}^{*}(t)\right)_{x}, p(t)\right\rangle_{V_{u^{*}}^{\prime}, V_{u^{*}}} d t
\end{aligned}
$$

which yields, by (34)-(36),

$$
\int_{Q}\left(-\lambda_{1} I_{f} u_{x}^{*}+\lambda_{3} I_{M}\right) Y d x d t+\lambda_{2} I_{T} \int_{0}^{L} Y(T, x) d x=\int_{Q} v y_{x}^{*} p_{x} d x d t .
$$

Comparing with (43) it follows that

$$
\int_{Q} v y_{x}^{*} p_{x} d x d t+\int_{Q} \lambda_{1} I_{f} v y_{x}^{*} d x d t \geq 0
$$

with $v=u-u^{*}$, for all $u \in U$, and this implies (33), as claimed. 


\subsection{Approximating problem}

In order to give a better characterization of the optimality condition (33) we determine an approximating form of it. To this end, for $\varepsilon>0$, we introduce an approximating problem $\left(P_{\varepsilon}\right)$ involving a nondegenerate state equation. The approximating optimality condition may be written more explicitly due to the better regularity of the approximating state and dual variable. Then we show that $\left(P_{\varepsilon}\right)$ tends in some sense to $(P)$. Namely, we show that a sequence of solutions to $\left(P_{\varepsilon}\right)$ tends to a solution to $(P)$, as $\varepsilon \rightarrow 0$.

We introduce

$$
\text { Minimize } J(u) \text { for all } u \in U_{\varepsilon},
$$

subject to the state system (11)-(仿), where

$$
\begin{aligned}
U_{\varepsilon} & =\left\{u \in W^{1, \infty}(0, L) ; u_{m}(x)+\varepsilon \leq u(x) \leq u_{M}(x)+2 \varepsilon,\right. \\
u(0) & \left.=u_{0}^{\varepsilon}, u(L)=u_{L}^{\varepsilon}, \quad\left|u_{x}(x)\right| \leq u_{\infty} \text { a.e. } x \in(0, L)\right\} .
\end{aligned}
$$

All hypotheses made in (21) -(23) remain the same and we note that if $u \in U_{\varepsilon}$, then

$$
\begin{gathered}
\varepsilon \leq u\left(x_{0}\right) \leq 2 \varepsilon \\
u_{m}(0)+\varepsilon \leq u_{0}^{\varepsilon} \leq u_{M}(0)+2 \varepsilon, \quad u_{m}(L)+\varepsilon \leq u_{L}^{\varepsilon} \leq u_{M}(L)+2 \varepsilon .
\end{gathered}
$$

For all $u \in U_{\varepsilon}, u(x) \geq u_{m}(x)+\varepsilon \geq \varepsilon$, and then system (11)-(3) with $u \in U_{\varepsilon}$ is nondegenerate.

By the general results concerning nondegenerate evolution equations in Hilbert spaces, if $y_{0} \in L^{2}(0, L)$, and $f \in L^{2}(Q)$, problem (1)-(3) with $u(x) \geq \varepsilon$ has a unique solution

$$
y_{\varepsilon} \in C\left([0, T] ; L^{2}(0, L)\right) \cap L^{2}\left(0, T ; H_{0}^{1}(0, L)\right) \cap W^{1,2}\left([0, T] ; H^{-1}(0, L)\right),
$$

(see [17], p. 163) and $y_{\varepsilon}$ satisfies the estimate

$$
\sup _{t \in[0, T]}\left\|y_{\varepsilon}(t)\right\|_{H}^{2}+\int_{0}^{T}\left\|\sqrt{u} y_{\varepsilon x}(t)\right\|_{H}^{2} d t \leq C,
$$

with $C$ a positive constant depending on the data and independent of $\varepsilon$.

We denote by $y_{\varepsilon x}$ the derivative of $y_{\varepsilon}$ with respect to $x$.

Obviously, the control problem $\left(P_{\varepsilon}\right)$ has at least a solution $\left(u_{\varepsilon}, y_{\varepsilon}\right)$, with $u_{\varepsilon} \in U_{\varepsilon}$ and $y_{\varepsilon}$ (corresponding to $u_{\varepsilon}$ ) satisfying (48). We prove the convergence result $\left(P_{\varepsilon}\right) \rightarrow(P)$ as $\varepsilon \rightarrow 0$, on the basis of the following lemma. 
Lemma 2.7. Let $y_{0} \in L^{2}(0, L), f \in L^{2}(Q), y_{0} \geq 0$ a.e. on $(0, L), f \geq 0$ a.e. on $Q$. Let $\left(u_{\varepsilon}, y_{\varepsilon}\right)$ be a sequence of solutions to $\left(P_{\varepsilon}\right)$ such that

$$
\begin{gathered}
u_{\varepsilon} \rightarrow u \text { uniformly in }[0, L] \text {, as } \varepsilon \rightarrow 0, \\
u_{\varepsilon x} \rightarrow u_{x} \text { weak }^{*} \text { in } L^{\infty}(0, L) \text {, as } \varepsilon \rightarrow 0 .
\end{gathered}
$$

Then, $u \in U$,

$$
\begin{gathered}
y_{\varepsilon} \rightarrow y \text { weakly in } L^{2}\left(0, T ; H_{u}^{1}(0, L)\right) \cap W^{1,2}\left([0, T] ;\left(H_{u}^{1}(0, L)\right)^{\prime}\right) \text {, as } \varepsilon \rightarrow 0, \\
y_{\varepsilon}(T) \rightarrow y(T) \text { weakly in } L^{2}(0, L) \text {, as } \varepsilon \rightarrow 0
\end{gathered}
$$

and $y$ is the solution to (1)-(3) corresponding to $u$.

Proof. It is easily seen that $\lim _{\varepsilon \rightarrow 0} u_{\varepsilon}=u \in U$ defined by (20). Then, $y_{\varepsilon}$ is the solution to the nondegenerate problem (11)-(3) corresponding to $u_{\varepsilon}$ and $y_{\varepsilon}$ satisfies (48). We get (on subsequences denoted still by the subscript $\varepsilon$ ) that

$$
\begin{gathered}
y_{\varepsilon} \rightarrow y \text { weakly in } L^{2}\left(0, T ; L^{2}(0, L)\right) \text {, as } \varepsilon \rightarrow 0, \\
\sqrt{u_{\varepsilon}} y_{\varepsilon} \rightarrow \xi \text { weakly in } L^{2}\left(0, T ; L^{2}(0, L)\right) \text {, as } \varepsilon \rightarrow 0, \\
y_{\varepsilon}(T) \rightarrow \zeta \text { weakly in } L^{2}(0, L), \text { as } \varepsilon \rightarrow 0 .
\end{gathered}
$$

We continue the proof in a similar way as in Theorem 2.5 and we get that $\xi=\sqrt{u} y$ a.e. on $Q$ and so $y \in L^{2}\left(0, T ; H_{u}^{1}(0, L)\right)$. By (14) we deduce that

$$
A y_{\varepsilon} \rightarrow A y \text { weakly in } L^{2}\left(0, T ;\left(H_{u}^{1}(0, L)\right)^{\prime}\right) \text {, as } \varepsilon \rightarrow 0
$$

and by (18)

$$
\frac{d y_{\varepsilon}}{d t} \rightarrow \frac{d y}{d t} \text { weakly in } L^{2}\left(0, T ;\left(H_{u}^{1}(0, L)\right)^{\prime}\right), \text { as } \varepsilon \rightarrow 0 .
$$

Following the arguments in Theorem 2.5 we prove (52) and that $y$ is the solution to (11)-(3) corresponding to $u$.

Theorem 2.8. Let $y_{0} \in L^{2}(0, L), f \in L^{2}(Q), y_{0} \geq 0$ a.e. on $(0, L)$, $f \geq 0$ a.e. on $Q$. Let $\left(u_{\varepsilon}^{*}, y_{\varepsilon}^{*}\right)_{\varepsilon>0}$ be a sequence of solutions to $\left(P_{\varepsilon}\right)$. Then (on subsequences denoted still by $\varepsilon_{\varepsilon}$ ) we have

$$
u_{\varepsilon}^{*} \rightarrow u^{*} \text { uniformly in }[0, L] \text {, as } \varepsilon \rightarrow 0,
$$




$$
u_{\varepsilon x}^{*} \rightarrow u_{x}^{*} \text { weak }{ }^{*} \text { in } L^{\infty}(0, L) \text {, as } \varepsilon \rightarrow 0,
$$

$y_{\varepsilon}^{*} \rightarrow y^{*}$ weakly in $L^{2}\left(0, T ; H_{u^{*}}^{1}(0, L)\right) \cap W^{1,2}\left([0, T] ;\left(H_{u^{*}}^{1}(0, L)\right)^{\prime}\right)$, as $\varepsilon \rightarrow 0$,

$$
y_{\varepsilon}(T) \rightarrow y(T) \text { weakly in } L^{2}(0, L) \text {, as } \varepsilon \rightarrow 0 .
$$

Moreover, $y^{*}$ is the solution to (11)-(3) corresponding to $u^{*}$ and $\left(u^{*}, y^{*}\right)$ is a solution to $(P)$.

Proof. Let $\left(u_{\varepsilon}^{*}, y_{\varepsilon}^{*}\right)$ be a solution to $\left(P_{\varepsilon}\right)$, i.e.,

$$
J\left(u_{\varepsilon}^{*}\right) \leq J\left(u_{\varepsilon}\right) \text {, for all } u_{\varepsilon} \in U_{\varepsilon} .
$$

Under the hypotheses for $y_{0}$ it follows that (1)-(3) with $u_{\varepsilon} \in U_{\varepsilon}$ has a unique solution $y_{\varepsilon} \in C\left([0, T] ; L^{2}(0, L)\right) \cap W^{1,2}\left([0, T] ;\left(H_{u_{\varepsilon}}^{1}(0, L)\right)^{\prime}\right) \cap L^{2}\left(0, T ; H_{u_{\varepsilon}}^{1}(0, L)\right)$ and

$$
\begin{aligned}
& \frac{\lambda_{1}}{2}\left(\int_{Q} u_{\varepsilon}^{*} y_{\varepsilon x}^{*} d x d t-M_{f}\right)^{2}+\frac{\lambda_{2}}{2}\left(\int_{0}^{L} y_{\varepsilon}^{*}(T, x) d x-M_{T}\right)^{2} \\
+ & \frac{\lambda_{3}}{2}\left(\int_{Q} y_{\varepsilon}^{*} d x d t-M\right)^{2} \\
\leq & \frac{\lambda_{1}}{2}\left(\int_{Q} u_{\varepsilon} y_{\varepsilon x} d x d t-M_{f}\right)^{2}+\frac{\lambda_{2}}{2}\left(\int_{0}^{L} y_{\varepsilon}(T, x) d x-M_{T}\right)^{2} \\
+ & \frac{\lambda_{3}}{2}\left(\int_{Q} y_{\varepsilon} d x d t-M\right)^{2}
\end{aligned}
$$

for all $u_{\varepsilon} \in U_{\varepsilon}$. Relations (153)-(154) follow from $u_{\varepsilon}^{*} \in U_{\varepsilon}$ and so the sequence of the corresponding states $\left(y_{\varepsilon}^{*}\right)_{\varepsilon}$ converges on a subsequence to $y^{*}$ the solution to (11)-(3) corresponding to $u^{*}$, as established by Lemma 2.7. In particular, we have (51) which implies that

$$
u_{\varepsilon} y_{\varepsilon x} \rightarrow u^{*} y_{x}^{*} \text { weakly in } L^{2}(Q), \text { as } \varepsilon \rightarrow 0 .
$$

Similarly, $u_{\varepsilon} \in U_{\varepsilon}$ implies that $u_{\varepsilon} \rightarrow u \in U$ uniformly on $[0, L]$ as $\varepsilon \rightarrow 0$, and by Lemma 2.7, (511)-(52), we get that $\left(y_{\varepsilon}\right)_{\varepsilon}$ is convergent to $y$ which is the solution to (11)-(3) corresponding to $u$. 
Passing to the limit in (57) we get

$$
\begin{gathered}
\frac{\lambda_{1}}{2}\left(\int_{Q} u^{*} y_{x}^{*} d x d t-M_{f}\right)^{2}+\frac{\lambda_{2}}{2}\left(\int_{0}^{L} y^{*}(T, x) d x-M_{T}\right)^{2}+\frac{\lambda_{3}}{2}\left(\int_{Q} y^{*} d x d t-M\right)^{2} \\
\leq \liminf _{\varepsilon \rightarrow 0}\left(\frac{\lambda_{1}}{2}\left(\int_{Q} u_{\varepsilon}^{*} y_{\varepsilon x}^{*} d x d t-M_{f}\right)^{2}+\frac{\lambda_{2}}{2}\left(\int_{0}^{L} y_{\varepsilon}^{*}(T, x) d x-M_{T}\right)^{2}\right. \\
\left.\quad+\frac{\lambda_{3}}{2}\left(\int_{Q} y_{\varepsilon}^{*} d x d t-M\right)^{2}\right) \\
\leq \limsup _{\varepsilon \rightarrow 0}\left(\frac{\lambda_{1}}{2}\left(\int_{Q} u_{\varepsilon} y_{\varepsilon x} d x d t-M_{f}\right)^{2}+\frac{\lambda_{2}}{2}\left(\int_{0}^{L} y_{\varepsilon}(T, x) d x-M_{T}\right)^{2}\right. \\
\left.\left.\leq \frac{\lambda_{1}}{2}\left(\int_{Q} u y_{x} d x d t-M_{f}\right)^{2}+\frac{\lambda_{2}}{2}\left(\int_{0}^{L} y(T, x) d x-M\right)^{2}\right)^{2}\right)^{2}+\frac{\lambda_{3}}{2}\left(\int_{Q} y d x d t-M\right)^{2}
\end{gathered}
$$

for all $u \in U$. This implies that $\left(u^{*}, y^{*}\right)$ is a solution to $(P)$.

\subsection{Approximating optimality conditions}

Let $K$ be a closed convex subset of a Banach space $X$ having the dual $X^{\prime}$.

We recall (see [2], p. 4-5) that the indicator function of $K$ is

$$
I_{K}(\xi)= \begin{cases}0, & \text { if } \xi \in K \\ +\infty, & \text { if } \xi \notin K\end{cases}
$$

and the subdifferential of $I_{K}$ coincides with the normal cone to $K$ at $\xi$,

$$
\partial I_{K}(\xi)=N_{K}(\xi)=\left\{\xi^{*} \in X^{\prime} ;\left\langle\xi^{*}, \xi\right\rangle_{X^{\prime}, X} \geq 0\right\} .
$$

For $\theta: \mathbb{R} \rightarrow[-1,1]$, let us denote by $\operatorname{sign} \theta$ the graph

$$
\operatorname{sign} \theta= \begin{cases}1, & \text { on }\{x ; \theta(x)>0\} \\ {[-1,1],} & \text { on }\{x ; \theta(x)=0\} \\ -1, & \text { on }\{x ; \theta(x)<0\}\end{cases}
$$


Proposition 2.9. Let us assume the hypotheses as in Theorem 2.8. Then, the approximating optimality condition reads as

$$
\int_{0}^{L}\left(u_{\varepsilon}^{*}-u_{\varepsilon}\right)(x) \Phi(x) d x \geq 0, \text { for all } u_{\varepsilon} \in U_{\varepsilon}
$$

where

$$
\Phi(x):=-\int_{0}^{T} y_{\varepsilon x}^{*}(t, x)\left\{p_{\varepsilon x}(t, x)+\lambda_{1}\left(\int_{Q} u_{\varepsilon}^{*} y_{\varepsilon x}^{*} d x d t-M_{f}\right)\right\} d t .
$$

Moreover, $\Phi$ has the representation

$$
\begin{aligned}
& \Phi(x)=-\rho^{\prime}(x)+\mu(x) \text { a.e. } x \in(0, L), \\
& u_{\varepsilon x}^{*}(x) \in u_{\infty} \operatorname{sign}(\rho(x)) \text { a.e. } x \in(0, L),
\end{aligned}
$$

where $\mu, \rho \in L^{1}(0, L)$,

$$
\left\{\begin{array}{l}
\mu(x) \leq 0 \text { a.e. in }\left\{x \in(0, L) ; u_{\varepsilon}^{*}(x)=u_{m}(x)+\varepsilon\right\} \\
\mu(x)=0 \text { a.e. in }\left\{x \in(0, L) ; u_{\varepsilon}^{*}(x) \in\left(u_{m}(x)+\varepsilon, u_{M}(x)+2 \varepsilon\right)\right\} \\
\mu(x) \geq 0 \text { a.e. in }\left\{x \in(0, L) ; u_{\varepsilon}^{*}(x)=u_{M}(x)+2 \varepsilon\right\},
\end{array}\right.
$$

and

$$
\rho(x)= \begin{cases}0 & \text { a.e. in }\left\{x \in(0, L) ;\left|u_{\varepsilon x}^{*}(x)\right|<u_{\infty} \text { a.e. }\right\} \\ \nu(x) u_{\varepsilon x}^{*}(x) & \text { a.e. in }\left\{x \in(0, L) ;\left|u_{\varepsilon x}^{*}(x)\right|=u_{\infty} \text { a.e. }\right\}\end{cases}
$$

with $\nu \in L^{1}(0, L), \nu \geq 0$ a.e. $x \in(0, L)$.

Proof. The system in variations, the dual system and the optimality conditions are similarly obtained as those for $(P)$. Namely, if $\left(u_{\varepsilon}^{*}, y_{\varepsilon}^{*}\right)$ is a solution to $\left(P_{\varepsilon}\right)$ we introduce

$$
\begin{gathered}
\frac{\partial p_{\varepsilon}}{\partial t}+\left(u_{\varepsilon}^{*} p_{\varepsilon x}\right)_{x} \\
=-\lambda_{1}\left(\int_{Q} u_{\varepsilon}^{*} y_{\varepsilon x}^{*} d x d t-M_{f}\right)+\lambda_{3}\left(\int_{Q} y_{\varepsilon}^{*} d x d t-M\right) \text { in } Q, \\
p_{\varepsilon}(T, x)=-\lambda_{2}\left(\int_{0}^{L} y_{\varepsilon}^{*}(T, x) d x-M_{T}\right) \text { in }(0, L), \\
p_{\varepsilon}(t, 0)=p_{\varepsilon}(t, L)=0 \text { in }(0, T),
\end{gathered}
$$


and deduce that $u_{\varepsilon}^{*}, y_{\varepsilon}^{*}$ and the dual variable $p_{\varepsilon}$ should satisfy a similar relation to (33), i.e.,

$$
\int_{Q}\left(u_{\varepsilon}^{*}-u_{\varepsilon}\right) y_{\varepsilon x}^{*}\left[p_{\varepsilon x}+\lambda_{1}\left(\int_{Q} u_{\varepsilon}^{*} y_{\varepsilon x}^{*} d x d t-M_{f}\right)\right] d x d t \leq 0
$$

for all $u_{\varepsilon} \in U_{\varepsilon}$. The solution $p_{\varepsilon}$ is regular, since $u_{\varepsilon}^{*} \geq \varepsilon$, and

$$
p_{\varepsilon} \in C\left([0, T] ; L^{2}(0, L)\right) \cap L^{2}\left(0, T ; H_{0}^{1}(0, L)\right) \cap W^{1,2}\left([0, T] ; H^{-1}(0, L)\right),
$$

by the same arguments as for $y_{\varepsilon}$ (see (47)).

The supplementary regularity of $y_{\varepsilon}$ and $p_{\varepsilon}$ implies that (67) can be still written

$$
\int_{0}^{L}\left(u_{\varepsilon}^{*}-u_{\varepsilon}\right)\left(\int_{0}^{T} y_{\varepsilon x}^{*}\left[p_{\varepsilon x}+\lambda_{1}\left(\int_{Q} u_{\varepsilon}^{*} y_{\varepsilon}^{*} d x d t-M_{f}\right)\right] d t\right) d x \leq 0,
$$

for all $u_{\varepsilon} \in U_{\varepsilon}$. We see that $\Phi \in L^{1}(0, L)$. Then, (58) and (59) imply that

$$
\Phi(x) \in N_{U_{\varepsilon}}\left(u_{\varepsilon}^{*}\right) .
$$

Here, $N_{U_{\varepsilon}}\left(u_{\varepsilon}^{*}\right)$ means the normal cone in $L^{1}(0, L)$ to $U_{\varepsilon}$ at $u_{\varepsilon}^{*} \in U_{\varepsilon}$, that is

$$
N_{U_{\varepsilon}}\left(u_{\varepsilon}^{*}\right)=\left\{w \in L^{1}(0, L) ; \int_{0}^{L}\left(u_{\varepsilon}^{*}-u_{\varepsilon}\right) w d x \geq 0, \text { for all } u_{\varepsilon} \in U_{\varepsilon}\right\} .
$$

We give further a representation of $N_{U_{\varepsilon}}\left(u_{\varepsilon}^{*}\right)$, more exactly we prove that any $w \in N_{U_{\varepsilon}}\left(u_{\varepsilon}^{*}\right)$ can be written in the form

$$
w=-\rho^{\prime}(x)+\mu(x) \text { a.e. } x \in(0, L)
$$

with $\rho$ and $\mu$ previously defined.

Indeed, if $w$ is given by (70) then

$$
\begin{aligned}
& \int_{0}^{L} w(x)\left(u_{\varepsilon}^{*}-u_{\varepsilon}\right) d x=\int_{0}^{L} \mu(x)\left(u_{\varepsilon}^{*}-u_{\varepsilon}\right) d x-\int_{0}^{L} \rho^{\prime}(x)\left(u_{\varepsilon}^{*}-u_{\varepsilon}\right) d x \\
= & \int_{0}^{L} \mu(x)\left(u_{\varepsilon}^{*}-u_{\varepsilon}\right) d x-\left.\rho\left(u_{\varepsilon}^{*}-u_{\varepsilon}\right)\right|_{0} ^{L}+\int_{0}^{L} \rho(x)\left(u_{\varepsilon x}^{*}-u_{\varepsilon x}\right) d x \geq 0
\end{aligned}
$$

for any $u_{\varepsilon} \in U_{\varepsilon}$, by (62) for the first term and (63) for the last term. This means that $w \in N_{U_{\varepsilon}}\left(u_{\varepsilon}^{*}\right)$. 
For the inverse implication we take $w \in N_{U_{\varepsilon}}\left(u_{\varepsilon}^{*}\right)$ and we note first that $U_{\varepsilon}$ can be written $U_{\varepsilon}=U_{1 \varepsilon}+U_{2 \varepsilon}$, where

$$
\begin{gathered}
U_{1 \varepsilon}=\left\{v \in W^{1, \infty}(0, L) ;\left|v_{x}\right| \leq u_{\infty} \text { a.e. } x \in(0, L), v(0)=u_{0}^{\varepsilon}, v(L)=u_{L}^{\varepsilon}\right\}, \\
U_{2 \varepsilon}=\left\{v \in L^{\infty}(0, L) ; u_{m}(x)+\varepsilon \leq v(x) \leq u_{M}(x)+2 \varepsilon \text { a.e. } x \in(0, L)\right\} .
\end{gathered}
$$

We also remark that $U_{1 \varepsilon} \cap$ int $U_{2 \varepsilon} \neq \varnothing$, and so

$$
\partial\left(I_{1}+I_{2}\right)=\partial I_{1}+\partial I_{2}
$$

(see [2], p. 7), where $I_{i}$ are the indicator functions of $U_{i \varepsilon}(i=1,2)$ and $\partial I_{i}$ denote their subdifferentials.

Therefore, $w \in N_{U_{\varepsilon}}\left(u_{\varepsilon}^{*}\right)=\partial I_{U_{\varepsilon}}\left(u_{\varepsilon}^{*}\right)$ is given by

$$
w=\xi+\mu, \xi \in \partial I_{1}\left(u_{\varepsilon}^{*}\right), \mu \in \partial I_{2}\left(u_{\varepsilon}^{*}\right) .
$$
(62).

It is obvious that $\mu(x) \in \partial I_{2}\left(u_{\varepsilon}^{*}\right)=N_{U_{2 \varepsilon}}\left(u_{\varepsilon}^{*}\right)$ if and only if $\mu(x)$ satisfies

The form of $\xi=-\rho^{\prime}$ (with $\rho$ given by (63) ) follows by some arguments based on general results given in [2], p. 11-15. For the reader's convenience we give a few details adapted to our case.

Let $\xi \in \partial I_{1}\left(u_{\varepsilon}^{*}\right)$ and $\gamma \in W^{1,1}(0, L)$ such that $\xi=-\gamma^{\prime}$ a.e. in $(0, L)$. This means that

$$
\xi=-\gamma^{\prime} \in \partial I_{1}\left(u_{\varepsilon}^{*}\right)
$$

and $\gamma \in \partial I_{F}\left(u_{\varepsilon x}^{*}\right)$, where $I_{F}$ is the indicator function of the set

$$
F=\left\{\zeta \in L^{\infty}(0, L) ; \zeta=v^{\prime} \text { a.e. in }(0, L), v \in U_{1 \varepsilon}\right\}
$$

Indeed, for all $v \in U_{1 \varepsilon}$, we have

$$
0 \leq \int_{0}^{L} \xi\left(u_{\varepsilon}^{*}-v\right) d x=\int_{0}^{L} \gamma\left(u_{\varepsilon x}^{*}-v_{x}\right) d x=\int_{0}^{L} \gamma\left(u_{\varepsilon x}^{*}-\zeta\right) d x, \text { for all } \zeta \in F .
$$

The set $F$ can be decomposed as $F_{1} \cap F_{2}$ where

$$
\begin{gathered}
F_{1}=\left\{\zeta \in L^{\infty}(0, L) ; \zeta=v^{\prime}, v \in W^{1, \infty}(0, L), v(0)=u_{0}^{\varepsilon}, v(L)=u_{L}^{\varepsilon}\right\} \\
F_{2}=\left\{\zeta \in L^{\infty}(0, L) ;|\zeta(x)| \leq u_{\infty} \text { a.e. } x \in(0, L)\right\} .
\end{gathered}
$$

Let us assume that there exists $w_{0} \in W^{1, \infty}(0, L)$ such that

$$
\left\|w_{0}\right\|_{L^{\infty}(0,1)}<u_{\infty}, w_{0}(0)=u_{0}^{\varepsilon}, w_{0}(L)=u_{L}^{\varepsilon} .
$$


We note that by (174), $w_{0} \in F_{1} \cap$ int $F_{2}$ and so

$$
\partial I_{F}=\partial I_{F_{1}}+\partial I_{F_{2}}
$$

(see again [2], p. 7). Therefore, $\gamma \in \partial I_{F}\left(u_{\varepsilon x}^{*}\right)$ can be written $\gamma=\gamma_{1}+\gamma_{2}$ with $\gamma_{i} \in \partial I_{F_{i}}\left(u_{\varepsilon x}^{*}\right)$. The subdifferential $\partial I_{F}$ is an application from $L^{\infty}(0, L)$ to $\left(L^{\infty}(0, L)\right)^{\prime}$ and so $\gamma_{i}$ are seen as elements belonging to $\left(L^{\infty}(0, L)\right)^{\prime}$, being represented as the sum of a continuous part $\gamma_{i a} \in L^{1}(0, L)$ and a singular part $\gamma_{i s}$ (see [2], p. 15). Then, $\gamma_{2 a} \in \partial I_{F_{2}}\left(u_{\varepsilon x}^{*}\right)$ a.e. $x \in(0, L)$ and by Proposition 1.9 in [2], p. 11-13, we have

$$
\gamma_{2 a}(x)= \begin{cases}0 & \text { a.e. in }\left\{x \in(0, L) ;\left|u_{\varepsilon x}^{*}(x)\right|<u_{\infty}\right\} \\ \nu(x) u_{\varepsilon x}^{*}(x) & \text { a.e. in }\left\{x \in(0, L) ;\left|u_{\varepsilon x}^{*}(x)\right|=u_{\infty}\right\}\end{cases}
$$

where $\nu \in L^{1}(0, L), \nu \geq 0$ a.e. in $(0, L)$.

Now, $\gamma_{1 a} \in \partial I_{F_{1}}\left(u_{\varepsilon x}^{*}\right)$ and so

$$
\int_{0}^{L} \gamma_{1 a}(x)\left(u_{\varepsilon x}^{*}-v_{x}\right)(x) d x=-\int_{0}^{L} \gamma_{1 a}^{\prime}(x)\left(u_{\varepsilon}^{*}-v\right)(x) d x \geq 0 \text {, for any } v \in F_{1} \text {. }
$$

In particular setting $v:=u_{\varepsilon}^{*}+l \phi$ with $\phi \in C_{0}^{\infty}(0, L)$ and $l>0$, we get

$$
\int_{0}^{L} \gamma_{1 a}^{\prime}(x) \phi(x) d x \geq 0 \text { for any } \phi \in C_{0}^{\infty}(0, L) .
$$

Setting $v:=u_{\varepsilon}^{*}-l \phi$ we get the inverse inequality and so it follows that

$$
\int_{0}^{L} \gamma_{1 a}^{\prime}(x) \phi(x) d x=0 \text { for any } \phi \in C_{0}^{\infty}(0, L),
$$

which implies that $\gamma_{1 a}^{\prime}(x)=0$.

In conclusion, $\gamma=\gamma_{1 a}+\gamma_{2 a}$, where $\left(\gamma_{1 a}\right)^{\prime}=0$ a.e. in $(0, L)$ and $\gamma_{2}:=\rho$ satisfies (75), so that by (73) we have $\xi=-\gamma^{\prime}=-\rho^{\prime}$. We note that

$$
-\rho^{\prime} \in \partial I_{1}\left(u_{\varepsilon}^{*}\right) \text { iff } \rho \in \partial I_{F}\left(u_{\varepsilon x}^{*}\right) .
$$

By (72) we get (70) and thus relation (60) follows from $\Phi(x) \in N_{U_{\varepsilon}}\left(u_{\varepsilon}^{*}\right)$.

On the subset $\left\{x \in(0, L) ;\left|u_{\varepsilon x}^{*}(x)\right|=u_{\infty}\right.$ a.e. $\}$ we have $\rho(x)=\nu(x) u_{\varepsilon x}^{*}(x)$ and we observe that there are two cases for $\nu>0$ :

$$
u_{\varepsilon x}^{*}(x)=\left\{\begin{aligned}
u_{\infty} & \text { if } u_{\varepsilon x}^{*}(x)=\frac{\rho(x)}{\nu(x)}>0 \\
-u_{\infty} & \text { if } u_{\varepsilon x}^{*}(x)=\frac{\rho(x)}{\nu(x)}<0 .
\end{aligned}\right.
$$

On the subset $\left\{x \in(0, L) ;\left|u_{\varepsilon x}^{*}(x)\right|<u_{\infty}\right.$ a.e. $\}$ the function $\rho(x)=0$. Therefore, we deduce (61), as claimed. 


\section{Homogeneous Dirichlet-Neumann bound- ary conditions}

In this section we consider the final time minimization problem

$$
\text { Minimize }\left(\int_{0}^{L} y^{u}(T, x) d x\right) \text { for all } u \in U,
$$

subject to

$$
\begin{gathered}
\frac{\partial y}{\partial t}-\left(u y_{x}\right)_{x}=f \text { in } Q, \\
y(0, x)=y_{0}(x) \text { in }(0, L), \\
y(t, 0)=0, y_{x}(t, L)=0 \text { in }(0, T) .
\end{gathered}
$$

The set $U$ and all hypotheses made for the functions occurring in $U$ are the same as in Section 2.2.

The existence of the solution to the new state system is treated by the variational technique, as in the case of the previous state system.

We introduce the space

$$
\begin{array}{r}
\widetilde{H_{u}^{1}}(0, L)=\left\{y \in L^{2}(0, L) ; y \text { locally absolutely continuous in }[0, L] \backslash\left\{x_{0}\right\},\right. \\
\left.\sqrt{u} y_{x} \in L^{2}(0, L), y(0)=0\right\},
\end{array}
$$

equipped with the scalar product

$$
(\theta, \bar{\theta})_{\widetilde{H_{u}^{1}(0, L)}}=\int_{0}^{L} \theta \bar{\theta} d x+\int_{0}^{L} u \theta_{x} \bar{\theta}_{x} d x, \text { for any } \theta, \bar{\theta} \in \widetilde{H_{u}^{1}}(0, L) .
$$

It is a Hilbert space with the norm given by (5) and with the dual denoted by $\left(\widetilde{H_{u}^{1}}(0, L)\right)^{\prime}$. We have the continuous and dense embeddings $\widetilde{H_{u}^{1}}(0, L) \hookrightarrow$ $L^{2}(0, L) \hookrightarrow\left(\widetilde{H_{u}^{1}}(0, L)\right)^{\prime}$.

For the subscripts we denote $\widetilde{V_{u}}=\widetilde{H_{u}^{1}}(0, L), \widetilde{V_{u}^{\prime}}=\left(\widetilde{H_{u}^{1}}(0, L)\right)^{\prime}$ and $H=$ $L^{2}(0, L)$.

The solution to (77)-(79) is defined according to Definition 2.3 by replacing $H_{u}^{1}(0, L)$ by $\widetilde{H_{u}^{1}}(0, L)$.

Let $u \in U$. We define $\widetilde{A}: \widetilde{H_{u}^{1}}(0, L) \rightarrow\left(\widetilde{H_{u}^{1}}(0, L)\right)^{\prime}$,

$$
\langle\widetilde{A} y, \psi\rangle_{\widetilde{V_{u}^{\prime}}, \widetilde{V_{u}}}=\int_{0}^{L} u y_{x} \psi_{x} d x, \text { for any } \psi \in \widetilde{H_{u}^{1}}(0, L) .
$$


Then, problem (77)-(79) has a unique solution

$$
y \in C\left([0, T] ; L^{2}(0, L)\right) \cap L^{2}\left(0, T ; \widetilde{H_{u}^{1}}(0, L)\right) \cap W^{1,2}\left([0, T] ;\left(\widetilde{H_{u}^{1}}(0, L)\right)^{\prime}\right)
$$

obtained by the first part of Theorem 2.4.

Theorem 3.1. Let $y_{0} \in L^{2}(0, L)$ and $f \in L^{2}(Q), y_{0} \geq 0$ a.e. on $(0, L)$, $f \geq 0$ a.e. on $Q$. Then $\left(P_{1}\right)$ has at least one solution.

The proof is led as in Theorem 2.5.

\subsection{Optimality conditions in the homogeneous Dirichlet- Neumann case}

Proposition 3.2. Let the assumptions of Proposition 2.9 hold. Assume in addition that

$$
\begin{gathered}
y_{0} \in H^{2}(0, L), f \in C\left([0, T] ; H^{1}(0, L)\right), y_{0 x}>0 \text { on }[0, L], f_{x}>0 \text { on } Q, \\
u_{M} \in W^{1, \infty}(0, L),\left\|u_{M}^{\prime}\right\|_{L^{\infty}(0, L)} \leq u_{\infty} .
\end{gathered}
$$

Then, $u^{*}$, a solution to $\left(P_{1}\right)$ has the form

$$
u^{*}(x)= \begin{cases}u_{0}+u_{\infty} x & \text { for } x \in\left[0, x_{1}\right) \\ u_{M}(x) & \text { for } x \in\left[x_{1}, x_{2}\right) \\ -u_{\infty}(x-L)+u_{L} & \text { for } x \in\left[x_{2}, L\right]\end{cases}
$$

where $x_{1}$ and $x_{2}$ are the solutions to

$$
u_{M}\left(x_{1}\right)=u_{\infty} x_{1}+u_{0}, u_{M}\left(x_{2}\right)=-u_{\infty}\left(x_{2}-L\right)+u_{L} .
$$

The function $u^{*}$ is unique for fixed $u_{M}, u_{m}, u_{0}, u_{L}, u_{\infty}$.

Proof. Since, in particular, $y_{0} \in L^{2}(0, L)$ and $f \in C(\bar{Q})$ it follows by Theorem 3.1 that $\left(P_{1}\right)$ has at least a solution $u^{*}$. We shall deduce the optimality condition for $\left(P_{1}\right)$ by passing to the limit in the approximating problem

$$
\text { Minimize }\left(\int_{0}^{L} y^{u}(T, x) d x\right) \text { for all } u \in U_{\varepsilon},
$$

subject to the state system (77) $-(79)$ with $U_{\varepsilon}$ given by (46) . 
All existence results and computations for the optimality condition in $\left(P_{1 \varepsilon}\right)$ are led similarly as for $\left(P_{\varepsilon}\right)$, but the dual system and the optimality condition are slightly modified due to the new cost functional and boundary conditions.

Let $\left(u_{\varepsilon}^{*}, y_{\varepsilon}^{*}\right)$ be a solution to $\left(P_{1 \varepsilon}\right)$. The new dual system is

$$
\begin{gathered}
\frac{\partial p_{\varepsilon}}{\partial t}+\left(u_{\varepsilon}^{*}(x) p_{\varepsilon x}\right)_{x}=0 \text { in } Q, \\
p_{\varepsilon}(T, x)=-1 \text { in }(0, L), \\
p_{\varepsilon}(t, 0)=p_{\varepsilon x}(t, L)=0 \text { in }(0, T),
\end{gathered}
$$

(here $\lambda_{1}=\lambda_{3}=0, \lambda_{2}=1$ ), and the optimality condition

$$
\int_{0}^{T} \int_{0}^{L}\left(u_{\varepsilon}^{*}-u_{\varepsilon}\right) y_{\varepsilon x}^{*} p_{\varepsilon x} d x d t \leq 0, \text { for all } u_{\varepsilon} \in U_{\varepsilon},
$$

is obtained by similar computations as for (67).

Since $y_{0} \in H^{2}(0, L), y_{\varepsilon}^{*}$ and $p_{\varepsilon x}$, solutions to nondegenerate equations, are more regular

$$
y_{\varepsilon}^{*}, p_{\varepsilon} \in C^{1}\left([0, T] ; L^{2}(0, L)\right) \cap C\left([0, T] ; H^{2}(0, L)\right),
$$

and in particular $y_{\varepsilon x}^{*}, p_{\varepsilon x} \in C([0, T] \times[0, L]), y_{\varepsilon x x}^{*}, p_{\varepsilon x x} \in C\left([0, T] ; L^{2}(0, L)\right)$. Then, we can write

$$
\int_{0}^{L}\left(u_{\varepsilon}^{*}-u_{\varepsilon}\right)\left(\int_{0}^{T} y_{\varepsilon x}^{*} p_{\varepsilon x} d t\right) d x \leq 0, \text { for all } u_{\varepsilon} \in U_{\varepsilon} .
$$

In addition, the convergence of $\left(P_{1 \varepsilon}\right)$ to $\left(P_{1}\right)$ as $\varepsilon \rightarrow 0$ follows as in Theorem 2.8 .

Moreover, according to Proposition 2.9 we have

$$
\begin{aligned}
& \widetilde{\Phi}(x)=-\rho^{\prime}(x)+\mu(x) \text { a.e. } x \in(0, L), \\
& u_{\varepsilon x}^{*}(x) \in u_{\infty} \operatorname{sign}(\rho(x)) \text { a.e. } x \in(0, L),
\end{aligned}
$$

where

$$
\widetilde{\Phi}(x):=-\int_{0}^{T} y_{\varepsilon x}^{*} p_{\varepsilon x} d t .
$$


We continue by establishing the sign of $\widetilde{\Phi}(x)$. The solution $y_{\varepsilon}^{*}$ is continuous and nonnegative on $[0, T] \times[0, L]$ and the minimum is attained on the boundary. Then by the strong maximum principle it follows that $y_{\varepsilon}^{*}>0$ on $(0, T) \times(0, L)$ and $-y_{\varepsilon x}^{*}(t, 0)<0$. Let $z=u_{\varepsilon}^{*} y_{\varepsilon x}^{*}$. Then, it satisfies

$$
\begin{aligned}
z_{t}-u_{\varepsilon}^{*} z_{x x}^{*} & =u_{\varepsilon}^{*} f_{x} \text { in } Q \\
z(0, x) & =u_{\varepsilon}^{*} y_{0 x} \text { in }(0, L), \\
z_{x}(t, 0) & >0, z_{x}(t, L)=0 \text { in }(0, T) .
\end{aligned}
$$

This nondegenerate problem has a unique solution, continuous and positive on $(0, T) \times(0, L)$, by the same strong maximum principle, hence $y_{\varepsilon x}^{*}(t, x)>0$, for any $(t, x) \in Q$.

From the dual problem we get $p_{\varepsilon} \leq 0$ on $[0, T] \times[0, L]$ and by a similar argument $-p_{\varepsilon x}(t, 0)>0$. If $\omega=u_{\varepsilon}^{*} p_{\varepsilon x}$ then $\omega$ satisfies

$$
\begin{aligned}
\omega_{t}+u_{\varepsilon}^{*} \omega_{x x}^{*} & =0 \text { in } Q, \\
\omega(0, x) & =0 \text { in }(0, L), \\
\omega_{x}(t, 0) & <0, \omega_{x}(t, L)=0 \text { in }(0, T)
\end{aligned}
$$

and it follows that $p_{\varepsilon x}(t, x)<0$ for any $(t, x) \in Q$.

In conclusion, $\widetilde{\Phi}(x)>0$ for all $x \in(0, L)$ and by (89) we note that

$$
\rho^{\prime}(x)=\mu(x)-\widetilde{\Phi}(x)
$$

preserves a negative sign only for $\mu(x) \leq 0$, i.e., on the subset

$$
U_{-}=\left\{x \in(0, L) ; u_{\varepsilon}^{*}(x)<u_{M}(x)+2 \varepsilon\right\} .
$$

It means that on this set $\rho(x) \neq 0$ except at most one point, and so we get by (90) that

$$
\left|u_{\varepsilon x}^{*}(x)\right|=u_{\infty} \text { a.e. in }\left\{x \in(0, L) ; u_{\varepsilon}^{*}(x)<u_{M}(x)+2 \varepsilon\right\},
$$

which is the eikonal equation. This solution cannot actually be uniquely determined by the conditions $u_{\varepsilon}^{*}(0)=u_{0}^{\varepsilon}, u_{\varepsilon}^{*}(L)=u_{L}^{\varepsilon}$, unless one observes, following a similar argument as in [4], that the function $u_{\varepsilon}^{*}$ is the maximal element of the set

$$
D=\left\{z \in W^{1, \infty}(0, L) ;\left|z^{\prime}(x)\right| \leq u_{\infty} \text { a.e. } x \in(0, L), z(x) \leq u_{\varepsilon}^{*} \forall x \in \partial U_{-}\right\}
$$


Here, $\partial U_{-}$is the boundary of $U_{-}$. To this end, let $z \in D$, and use (89) to get on the right-hand that

$$
\begin{aligned}
& \int_{U_{-}} \rho^{\prime}(x)\left(u_{\varepsilon}^{*}(x)-z(x)\right)^{-} d x \\
= & \int_{U_{-}} \mu(x)\left(u_{\varepsilon}^{*}(x)-z(x)\right)^{-} d x-\int_{U_{-}} \widetilde{\Phi}(x)\left(u_{\varepsilon}^{*}(x)-z(x)\right)^{-} d x \leq 0 .
\end{aligned}
$$

On the other hand

$$
\begin{aligned}
& \int_{U_{-}} \rho^{\prime}(x)\left(u_{\varepsilon}^{*}(x)-z(x)\right)^{-} d x \\
= & \left.\rho(x)\left(u_{\varepsilon}^{*}(x)-z(x)\right)^{-}\right|_{\partial U_{-}}+\int_{U_{-}} \rho(x)\left(u_{\varepsilon x}^{*}(x)-z_{x}(x)\right) d x \geq 0 .
\end{aligned}
$$

Since $\rho^{\prime}<0$ on $U_{-}$, we necessarily obtain that $\left(u_{\varepsilon}^{*}(x)-z(x)\right)^{-}=0$, meaning that $z(x) \leq u_{\varepsilon}^{*}(x)$ for any $z \in D$.

In conclusion, $u_{\varepsilon}^{*}(x)$ must have the slope equal to either $u_{\infty}$ or $-u_{\infty}$ in the subset where $u_{\varepsilon}^{*}(x)<u_{M}+2 \varepsilon$, and being the maximal element of $D$, it is unique. In the subset where $u_{\varepsilon}^{*}(x)=u_{M}(x)+2 \varepsilon$ its slope is $u_{M}^{\prime} \in\left(-u_{\infty}, u_{\infty}\right)$. Therefore, for given data $u_{M}, u_{m}, u_{0}^{\varepsilon}, u_{L}^{\varepsilon}, u_{\infty}$, the possible representation of $u_{\varepsilon}^{*}$ is

$$
u_{\varepsilon}^{*}(x)= \begin{cases}u_{0}^{\varepsilon}+u_{\infty} x & \text { for } x \in\left[0, x_{1}^{\varepsilon}\right) \\ u_{M}(x)+2 \varepsilon & \text { for } x \in\left[x_{1}^{\varepsilon}, x_{2}^{\varepsilon}\right) \\ -u_{\infty}(x-L)+u_{L}^{\varepsilon} & \text { for } x \in\left[x_{2}^{\varepsilon}, L\right],\end{cases}
$$

where $x_{1}^{\varepsilon}$ is the abscissa of the intersection of the graphic of the function $x \rightarrow u_{M}(x)+2 \varepsilon$ with the line of slope $u_{\infty}$ passing through $u_{0}^{\varepsilon}$, and $x_{2}^{\varepsilon}$ is the abscissa of the intersection of the graphic of the function $x \rightarrow u_{M}(x)+2 \varepsilon$ with the line of slope $u_{\infty}$ passing through $u_{L}^{\varepsilon}$, i.e.,

$$
u_{M}\left(x_{1}^{\varepsilon}\right)+2 \varepsilon=u_{\infty} x_{1}^{\varepsilon}+u_{0}^{\varepsilon}, u_{M}\left(x_{2}^{\varepsilon}\right)+2 \varepsilon=-u_{\infty}\left(x_{2}^{\varepsilon}-L\right)+u_{L}^{\varepsilon} .
$$

Passing to the limit in (91) as $\varepsilon \rightarrow 0$, on the basis of the convergence of $\left(P_{1 \varepsilon}\right)$ to $\left(P_{1}\right)$ we get $(\underline{83})$.

As an example, the feature of a possible $u^{*}$ given by (83) is seen in Fig. 3 , by a solid line. It was computed for

$$
\begin{aligned}
L & =1, T=2, x_{0}=0.7, \\
u_{M}(x) & =20\left(x-x_{0}\right)^{2}, u_{m}(x)=\left(x-x_{0}\right)^{2}, \\
u_{0} & =3, u_{L}=1, u_{\infty}=10 .
\end{aligned}
$$


The graphic of $u_{M}$ is drawn by a dashed line.

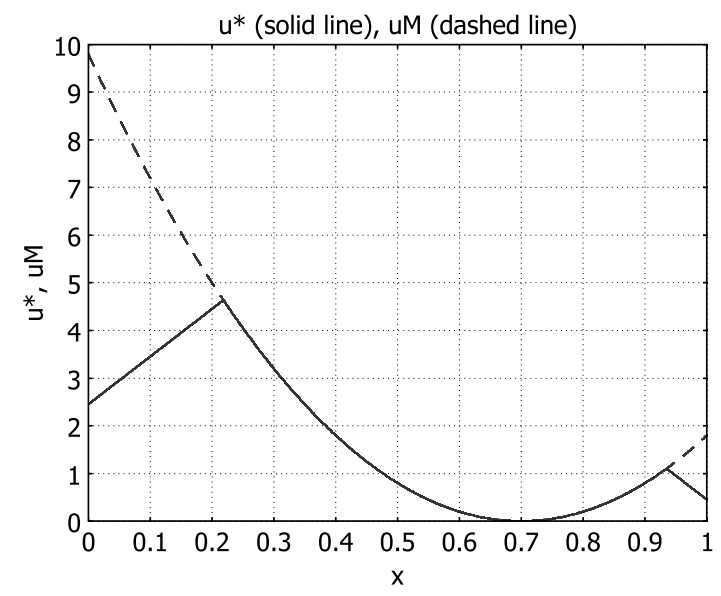

Fig. 3. $u^{*}(x)$ given by (83) and $u_{M}(x)$

\section{Numerical results}

We note that the form of $u^{*}$ depends on the restrictions included in the set $U$, namely on the choice of $u_{M}, u_{m}, u_{\infty}, u_{0}$ and $u_{L}$, the greater importance being that of $u_{M}$. We present some numerical simulations to put into evidence the influence of $u_{M}$ in the case when it is a polynomial function. The data are

$$
\begin{aligned}
L & =10, T=0.5, x_{0}=5, y_{0}(x)=\sin (4 x)+|\sin (4 x)|, f=0, \\
u_{M}(x) & =2\left|x-x_{0}\right|^{n}, u_{m}(x)=\left|x-x_{0}\right|^{n} \\
u_{\infty} & =10, u_{0}=\left(u_{M}(0)+u_{m}(0)\right) / 2, u_{L}=\left(u_{M}(L)+u_{m}(L)\right) / 2
\end{aligned}
$$

and $n=3$ (Fig. 4.1, 4.2), $n=2$ (Fig. 5.1, 5.2), $n=1$ (Fig. 6.1, 6.2). The figures represent the graphics of $u^{*}(x)$ computed by (83) and the graphics of $y^{*}(T, x)$ representing the corresponding solution to (77)-(79) at $T=0.5$. All computations are done with Comsol Multiphysics v. 3.5a (FLN License 1025226).

The graphics of $y^{*}(T, x)$ in Figs. 4.2, 5.2, 6.2 and the values $I=\int_{0}^{L} y^{*}(T, x) d x$ indicate $n=3$ as the best value because $I$ is minimum in this case. The practice of the various physical processes to which these results can be applied can indicate the choice of $u_{M}(x)$ and $u_{m}(x)$ in classes of functions other than polynomial, and this may lead to better results. 


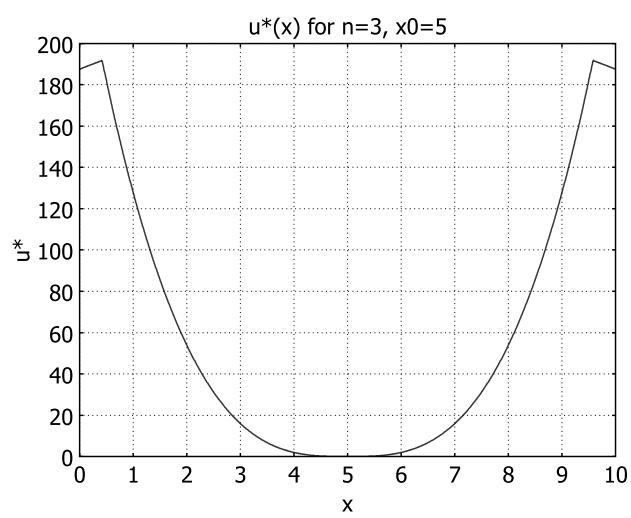

Fig. 4.1. $u^{*}(x)$ for $n=3$

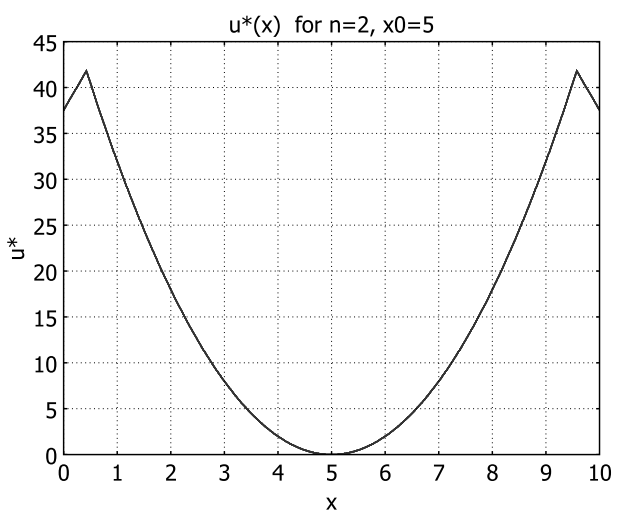

Fig. 5.1. $u^{*}(x)$ for $n=2$

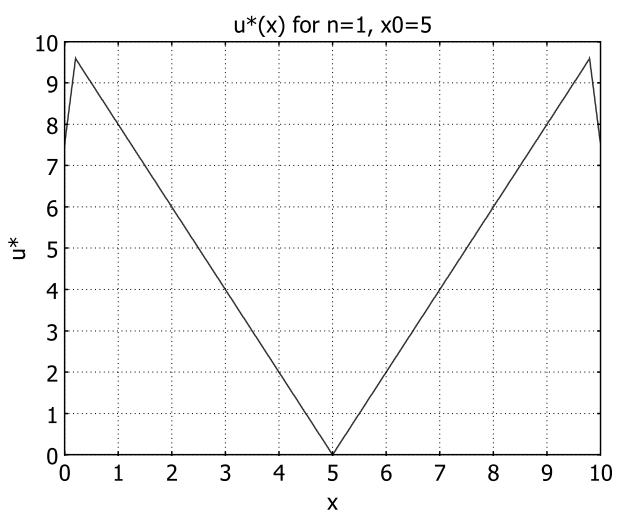

Fig. 6.1. $u^{*}(x)$ for $n=1$

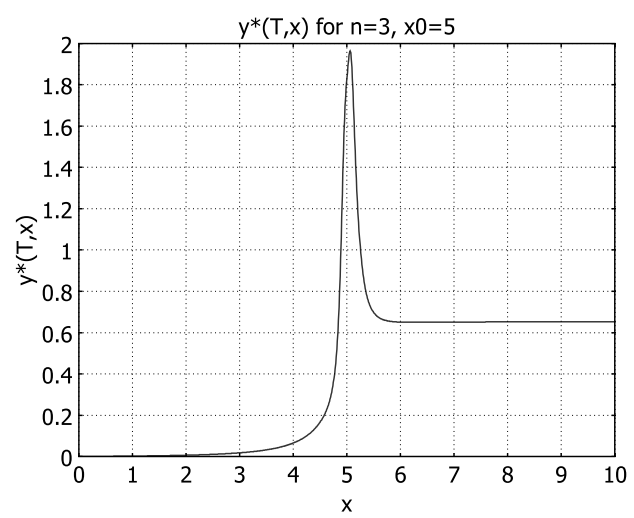

Fig. 4.2. $y^{*}(T, x)$ for $n=3$

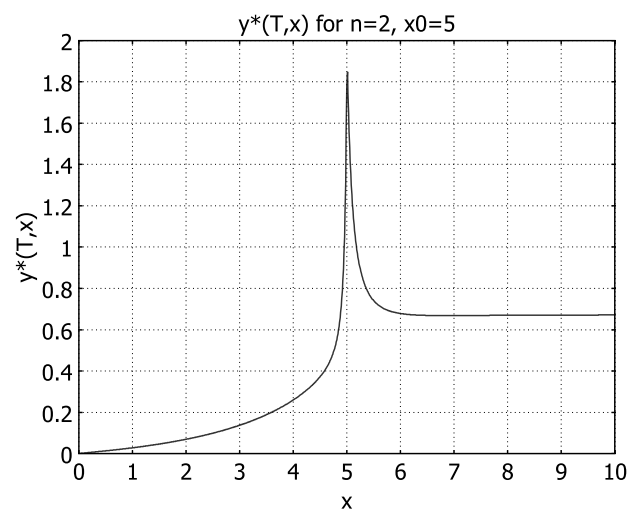

Fig. 5.2. $y^{*}(T, x)$ for $n=2$

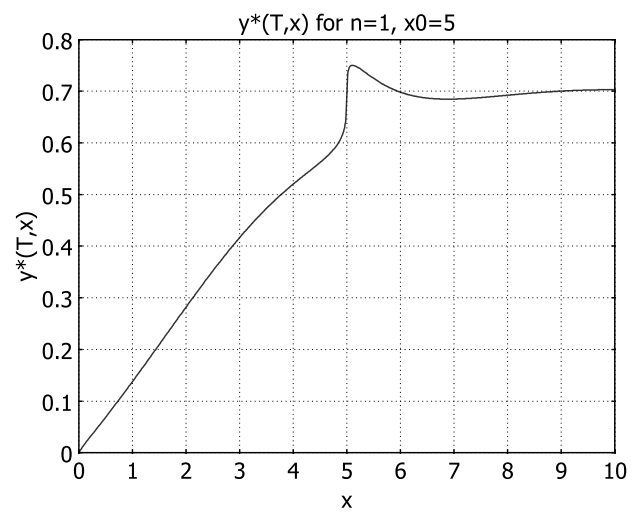

Fig. 6.2. $y^{*}(T, x)$ for $n=1$ 
Acknowledgments. G.M. acknowledges the support of INDAM-GNAMPA, Italy, for May-June 2013 and of the grant CNCS-UEFISCDI, project number PN-II-ID-PCE-2011-3-0027. G.F., R.M.M., S.R. acknowledge the support of the GNAMPA project "Equazioni di evoluzione degeneri e singolari: controllo e applicazioni", 2013.

\section{References}

[1] Alabau-Boussouira F, Cannarsa P and Fragnelli G 2006 Carleman estimates for degenerate parabolic operators with applications to null controllability J. Evol. Equ. 6 161-204

[2] Barbu V 1994 Mathematical Methods in Optimization of Differential Systems (Dordrecht: Kluwer Academic Publishers)

[3] Barbu V 2010 Nonlinear Differential Equations of Monotone Types in Banach Spaces (New York: Springer)

[4] Barbu V and Friedman A 1991 Optimal design of domains with free boundary problems SIAM J. Control Optimiz. 29 623-627

[5] Belmiloudi A 2005 Nonlinear optimal control problems of degenerate parabolic equations with logistic time-varying delays of convolution type Nonlinear Anal. 63 1126-1152

[6] Buchot J M and Raymond J P 2002 A linearized model for boundary layer equations in Optimal control of complex structures (Oberwolfach 2000) Internat. Ser. Numer. Math. 139 (Basel: Birkhäuser) 31-42

[7] Caldiroli P and Musina R 2000 On a variational degenerate elliptic problem NoDEA Nonlinear Differ. Equ. Appl. 7 187-199

[8] Campiti M, Metafune G and Pallara D 1998 Degenerate self-adjoint evolution equations on the unit interval Semigroup Forum 57 1-36

[9] Cannarsa P, Tort J and Yamamoto M 2010 Determination of source terms in a degenerate parabolic equation Inverse Problems 26 1-20

[10] Favini A and Marinoschi G 2010 Identification of the time derivative coefficient in a fast diffusion degenerate equation J. Optim. Theory Appl. 145 249-269 
[11] Favini A and Yagi A 1999 Degenerate Differential Equations in Banach Spaces (New York: Marcel Dekker Inc.)

[12] Fleming W H and Viot M 1979 Some measure-valued Markov processes in population genetics theory Indiana Univ. Math. J. 28 817-843

[13] Fragnelli G, Ruiz Goldstein G, Goldstein J A and Romanelli S 2012 Generators with interior degeneracy on spaces of $L^{2}$ type Electron. J. Differential Equations 2012 1-30

[14] Fragnelli G and Mugnai D Carleman estimates and observability inequalities for parabolic equations with interior degeneracy (submitted)

[15] Goldstein J A and Lin A Y 1991 An $L^{p}$ semigroup approach to degenerate parabolic boundary value problems Ann. Mat. Pura Appl. 4 211-227

[16] Lenhart S and Yong J M 1995 Optimal control for degenerate parabolic equations with logistic growth Nonlinear Anal. 25 681-698

[17] Lions J L 1969 Quelques méthodes de resolution des problèmes aux limites non linéaires (Paris: Dunod)

[18] Marinoschi G 2009 Optimal control of metabolite transport across cell membranes driven by the membrane potential Nonlinear Anal. Real World Appl. $101276-1298$

[19] Mininni R M and Romanelli S 2003 Martingale estimating functions for Feller diffusion processes generated by degenerate elliptic operators J. Concr. Appl. Math. 1 191-216

[20] Stahel A 1992 Degenerate semilinear parabolic equations Differential Integral Equations 5 683-691

[21] Shimakura N 1992 Partial Differential Operators of elliptic type Translations of Mathematical Monographs 99 (Providence: American Mathematical Society)

[22] Tort J and Vancostenoble J 2012 Determination of the insolation function in the nonlinear Sellers climate model Ann. I. H. Poincaré-AN 29 683-713

[23] Vespri V 1989 Analytic semigroups, degenerate elliptic operators and applications to nonlinear Cauchy problems Ann. Mat. Pura Appl. (IV) CLV $353-388$ 